\title{
Towards Neuromorphic Photonic Networks of Ultrafast Spiking Laser Neurons
}

\author{
J. Robertson, E. Wade, Y. Kopp, J. Bueno and A. Hurtado*
}

\begin{abstract}
We report on ultrafast artificial laser neurons and on their potentials for future neuromorphic (brain-like) photonic information processing systems. We introduce our recent and ongoing activities demonstrating controllable excitation of spiking signals in optical neurons based upon Vertical-Cavity Surface Emitting Lasers (VCSEL-Neurons). These spiking regimes are analogous to those exhibited by biological neurons, but at sub-nanosecond speeds ( $>7$ orders of magnitude faster). We also describe diverse approaches, based on optical or electronic excitation techniques, for the activation/inhibition of sub-ns spiking signals in VCSEL-Neurons. We report our work demonstrating the communication of spiking patterns between VCSEL-Neurons towards future implementations of optical neuromorphic networks. Furthermore, new findings show that VCSEL-Neurons can perform multiple neuro-inspired spike processing tasks. We experimentally demonstrate photonic spiking memory modules using single and mutually-coupled VCSEL-Neurons. Additionally, the ultrafast emulation of neuronal circuits in the retina using VCSEL-Neuron systems is demonstrated experimentally for the first time to our knowledge. Our results are obtained with off-the-shelf VCSELs operating at the telecom wavelengths of 1310 and $1550 \mathrm{~nm}$. This makes our approach fully compatible with current optical network and data centre technologies; hence offering great potentials for future ultrafast neuromorphic laser-neuron networks for new paradigms in brain-inspired computing and Artificial Intelligence.
\end{abstract}

Index Terms - Neuromorphic Photonics, VCSELs, Nonlinear Dynamics, Photonic Spiking, Neuronal Networks.

\section{INTRODUCTION}

$\mathrm{N}$ EUROMORPHIC computing systems aim at carrying out information processing tasks inspired by the brain's powerful computational capabilities. Electronic approaches for artificial neuronal models have been studied for decades [1]. Technology has greatly matured and multiple neuromorphic computing platforms based on electronic circuitry techniques have been developed [2-5], e.g. Stanford's Neurogrid [2],

Manuscript received April 5, 2019. This work was supported by the Office of Naval Research Global (Grant ONRGNICOP-N62909-18-1-2027), the European Commission, (Grant 828841-ChipAI-H2020-FETOPEN-2018-2020 and the EPSRC Doctoral Training Partnership (EP/N509760).

The authors are with the Institute of Photonics, Dept. Physics, University of Strathclyde, Technology and Innovation Centre, 99 George Street, G1 1RD, Glasgow (Scotland, UK) (antonio.hurtado@strath.ac.uk).

All data underpinning this publication are openly available from the University of Strathclyde KnowledgeBase at

https://doi.org/10.15129/6e27fda2-3a26-4dfd-8314-a242680de2de
HICANN at the University of Heidelberg [3], TrueNorth at IBM [4] and SpiNNaker at the University of Manchester [5]. These have demonstrated impressive performance levels allowing simultaneous operation of thousands of interconnected artificial neurons. However, neuromorphic electronic approaches have inherent limitations (e.g. reduced bandwidths and operation speeds, limited lengths of communication links, crosstalk, etc.) affecting system operation. Consequently, since the turn of the millennia [6], photonic approaches for neuromorphic systems benefiting from the unique properties of light-enabled technologies (e.g. ultrafast speeds, large bandwidths, low crosstalk, large communication links, wavelength multiplexing, large integration, etc.) have emerged. These offer great promise for a new generation of brain-inspired computing platforms with enhanced performance and the ability to operate up to 9 (up to 6) orders of magnitude faster than the biological (electronic artificial) neurons [7]. Whilst, reports on neuromorphic photonic approaches started to appear slowly but consistently at the beginning of 2010 and over the past decade the field has exploded with the toolkit of neuromorphic photonic technologies greatly expanding. These include systems based upon photonic crystal structures [8,9], resonant tunneling diode-laser diode (RTD-LD) coupled systems [10,11], fibre lasers [12,13], semiconductor optical amplifiers (SOAs) $[14,15]$, optical modulators [16,17] and semiconductor lasers (SLs) [18-49]. Amongst those, approaches based on SLs have dominated, as these devices exhibit multiple behaviors also observed in neurons, such as excitability [29,50-53] and complex non-linear dynamics $[54,55]$. Therefore, a rich diversity of artificial optical neurons based on different types of SLs have appeared. These include systems using micro-disk [18], micro-pillar [19-21] and micro-ring lasers [22-23], quantum dot (QD) lasers [24-26], two-section lasers with saturable absorber regions [27] and vertical-cavity surfaceemitting lasers (VCSELs) [28-32,35-47]. Further details can be found in recent reviews $[7,33,34]$ and references therein.

Specifically, approaches for artificial optical neurons based on VCSELs are receiving increasing research interest, given the interesting attributes of these devices which make them ideal for their future deployment in highly interconnected neuromorphic laser neuron networks. Among the inherent advantages of VCSELs we can cite for example: ultra-small footprint, low cost, high-speed, potentials for large scale integration into complex architectures including $2 \mathrm{D} / 3 \mathrm{D}$ arrays, operation at telecom wavelengths, etc. [56,57]. The use of 
VCSELs as artificial neuronal models (referred from now onwards as VCSEL-Neurons) was proposed as early as 2010 [35] using different techniques for operation, such as polarization switching (PS) [36] and optical injection (OI) induced nonlinear dynamics [54,55]. Since then, multiple reports on VCSELs for ultrafast spiking photonic neuronal models have emerged [28-32][45-57]. Moreover, VCSELNeurons operating at both short (e.g. $850 \mathrm{~nm})$ [29-31] and long-wavelengths $(1310 \mathrm{~nm}, 1550 \mathrm{~nm})$ have been experimentally demonstrated [28][37-41]. Specifically, using short-wavelength VCSEL-Neurons, the activation, storing and control of excitable spikes under phase-modulated optical injection has been reported [29-31]. Our research group has strategically focused on neuromorphic systems with VCSELNeurons at longer wavelengths in the telecom windows of $1310 \mathrm{~nm}$ and $1550 \mathrm{~nm}$; hence making our approaches fully compatible with current optical telecommunication networks and data center technologies. Recently, we have experimentally demonstrated the controllable excitation [37,38], and inhibition [39], of sub-ns excitable spike patterns replicating various biological neuronal responses. The latter includes spike thresholding (requirement of a super-threshold stimulus strength for the system to fire a spiking response), phasic and tonic spiking (single or continuous spike firing following the arrival of a stimulus), spike latency and spike firing rate encoding (the ability to encode stimulus strength information in the latency of spike firing or in the interspiking firing interval), etc. Table 1 summarizes the different techniques used to develop and investigate VCSEL-Neurons.

TABLE I

TECHNIQUES FOR ARTIFICIAL OPTICAL NEURONS WITH VCSELS

\begin{tabular}{|c|c|c|}
\hline Technique & Wavelength & $\begin{array}{l}\text { Experimental/ } \\
\text { Numerical }\end{array}$ \\
\hline $\begin{array}{c}\text { Spike activation via polarization } \\
\text { switching }[28,35]\end{array}$ & $1550 \mathrm{~nm}$ & Experimental \\
\hline $\begin{array}{c}\text { Spike activation via electrical bias } \\
\text { stimulation [38] }\end{array}$ & $1310 \mathrm{~nm}$ & Experimental \\
\hline $\begin{array}{l}\text { Spike activation via phase-modulated } \\
\text { optical injection [29-31] }\end{array}$ & $980 \mathrm{~nm}$ & $\begin{array}{l}\text { Experimental/ } \\
\text { Numerical }\end{array}$ \\
\hline $\begin{array}{c}\text { Spike activation via amplitude- } \\
\text { modulated optical injection }[37,43-44]\end{array}$ & $1310 \mathrm{~nm}$ & $\begin{array}{l}\text { Experimental/ } \\
\text { Numerical }\end{array}$ \\
\hline $\begin{array}{l}\text { Spike inhibition via amplitude- } \\
\text { modulated optical injection }[39,43]\end{array}$ & $1310 \mathrm{~nm}$ & $\begin{array}{l}\text { Experimental/ } \\
\text { Numerical }\end{array}$ \\
\hline $\begin{array}{l}\text { Spike activation/inhibition via saturable } \\
\text { absorber region }[27,47-48]\end{array}$ & $850 \mathrm{~nm}$ & Numerical \\
\hline $\begin{array}{c}\text { Networked/coupled spiking VCSELs } \\
{[40-42,44]}\end{array}$ & $1310 \mathrm{~nm}$ & $\begin{array}{l}\text { Experimental/ } \\
\text { Numerical }\end{array}$ \\
\hline
\end{tabular}

Nevertheless, to date the majority of works on SL-based photonic spiking neurons have focused on single devices. Yet, it is widely acknowledged that the implementation of interconnected architectures is needed to develop neuromorphic photonic systems for use in practical computing and Artificial Intelligence applications. Therefore, we have focused on the connectivity between different VCSELNeurons and have demonstrated the successful communication of excitatory [40] and inhibitory [41] spiking signals between coupled VCSEL-Neurons at telecom wavelengths. Also, an interesting work has very recently outlined the use of coupled VCSEL-arrays (at short-wavelengths) for use in neuromorphic applications [42]. Additionally, other works have numerically investigated different interconnectivity architectures between coupled VCSEL-Neurons (at telecom wavelengths) using PS for operation [43-45]. Further, theoretical works have also recently described the potentials of photonic neuronal models based on VCSELs with a saturable absorbing region in their structure and VCSELs in combination with vertical cavity semiconductor optical amplifiers, for different spiking processing tasks, including spiking memory, spike encoding, spike timing dependent plasticity and pattern recognition [27,46-49].

In this work we introduce our recent and ongoing research work on ultrafast VCSEL-Neuron systems for neuromorphic photonic applications. We first review (in Section II) our work on ultrafast photonic spiking regimes in VCSEL-Neurons at telecom wavelengths through both previously demonstrated experiments (Figs. 2-8,11-13 [37-40]) and new findings (Figs. $9-10)$. We describe our experimental results demonstrating the controllable and reproducible excitation and inhibition of photonic spiking regimes at sub-ns speed rates in VCSELNeurons under different optical and electronic excitation schemes. We also introduce (in Section II) our activities demonstrating the successful communication of sub-ns photonic spiking regimes between interconnected VCSELNeurons towards future network realizations. Section III reports new experimental results that demonstrate the use of VCSEL-Neuron systems to perform photonic spiking processing tasks at sub-ns speeds. We introduce our experimental work on photonic spiking memory platforms using two different architectures, namely a single VCSELNeuron subject to delayed optical feedback, and two mutually coupled VCSEL-Neurons. We demonstrate that complex spiking patterns, with sub-ns temporal spike lengths, can be stored in each of the system architectures studied. Section III also introduces our current experimental work on the ultrafast photonic emulation of biological neuronal circuits with VCSEL-Neurons. In particular, we report our experimental results demonstrating the photonic emulation of neuronal circuits in the human retina, involving different types of spiking and non-spiking neurons. Finally, Section IV collects the conclusions and future lines of action for this work.

\section{II.UltRAFAST SPIKING REgIMES IN VCSEL-NEURONS}

This section presents our research work on the controllable and reproducible excitation, inhibition and communication of photonic spiking regimes at ultrafast sub-ns speed rates using VCSEL-Neurons. It is important to note that all experimental results in this work have been obtained with commerciallyavailable VCSELs operating at telecom wavelengths. Fig. 1 plots typical L-I curves (Fig. 1(a)) and spectra (Figs. 1(b,c)) of devices used in this work at the $1310 \mathrm{~nm}$ and $1550 \mathrm{~nm}$ windows. The measured threshold currents ranged from 0.6 $\mathrm{mA}$ to $1.8 \mathrm{~mA}$ at room temperature. Also, for both wavelength windows, the devices' spectra showed two peaks corresponding to the two orthogonal polarizations of the device's fundamental transverse mode. These are referred as parallel $\left(\lambda_{\mathrm{y}}\right)$ and orthogonally $\left(\lambda_{\mathrm{x}}\right)$ polarized modes. The 
spectra also show that one of the two modes dominates (lasing mode) and the other one remains suppressed (subsidiary mode). For the cases included in Fig. 1, the main lasing modes were the parallel and the orthogonally-polarized modes respectively for the devices operating at $1310 \mathrm{~nm}$ (Fig. 1(a)) and at $1550 \mathrm{~nm}$ (Fig. 1(b)). All VCSELs used in this work exhibited similar properties with wavelength separations of $\sim 0.3 \mathrm{~nm}$ between the two supported modes.
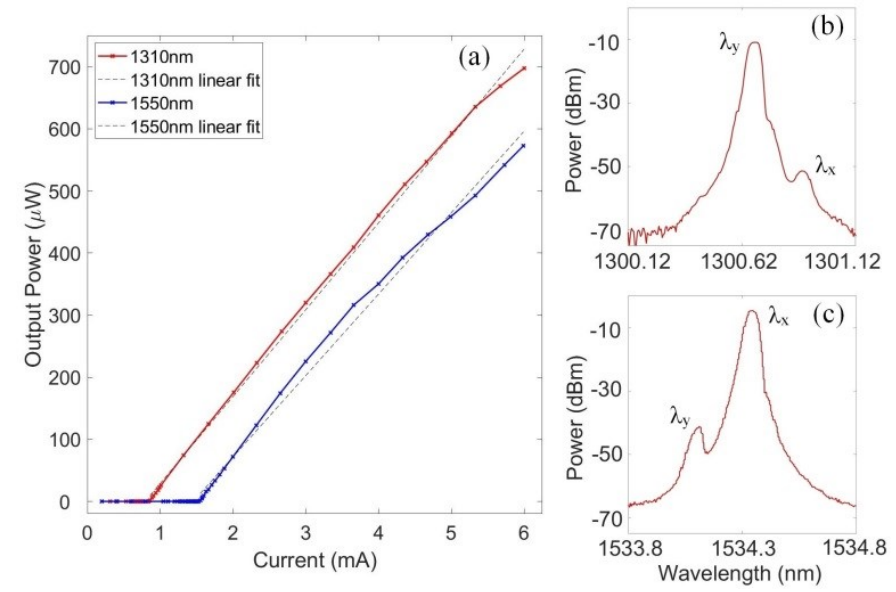

Fig. 1. Typical L-I curve (a) and optical spectra (b \& c) of the VCSELs used in this work to investigate their use in neuromorphic photonic systems. Devices operating at the wavelengths of (b) $1310 \mathrm{~nm}$ and (c) $1550 \mathrm{~nm}$ were used. The devices' two polarization modes are referred to as $\lambda_{y}$ (parallel) and $\lambda_{\mathrm{x}}$ (orthogonal).

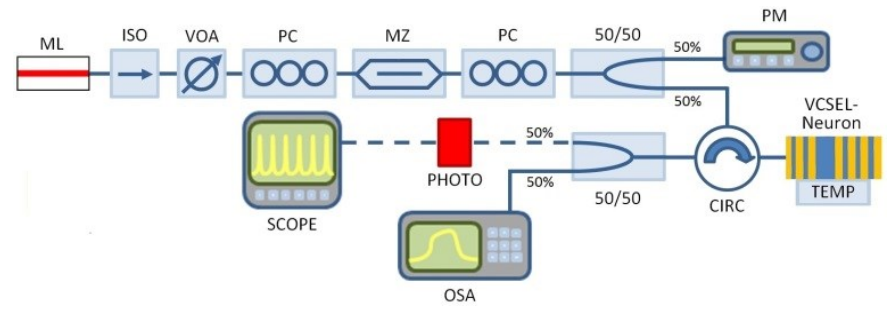

Fig. 2. Setup used to activate/inhibit spiking dynamics at sub-ns rates in VCSEL-neurons. ML-master laser, ISO-optical isolator, VOA-variable optical attenuator, PC-polarization controller, MZ-Mach-Zehnder amplitude modulator, PM-power meter, CIRC-circulator, TEMP-temperature controls, PHOTO-photodetector, OSA-optical spectrum analyser, SCOPE-oscilloscope.

\section{A. Generation of Spiking Regimes in VCSEL-Neurons}

We introduce first the previously reported technique that lays the foundation for our work on the excitation of controllable and reproducible spiking patterns using VCSELNeurons operating at telecom wavelengths. Fig. 2 plots the experimental setup where a VCSEL (either at $1310 \mathrm{~nm}$ or $1550 \mathrm{~nm}$ ) is used as an artificial optical neuron under the arrival of incoming stimuli (perturbations) from an externallymodulated tunable laser source (for full details see [37,39]). The external signals with the encoded stimuli from the tunable laser were injected into the VCSEL-neuron via an optical circulator. The latter also collected the outputs for analysis with a fast $(13 \mathrm{GHz})$ real time oscilloscope and an optical spectrum analyzer (OSA).

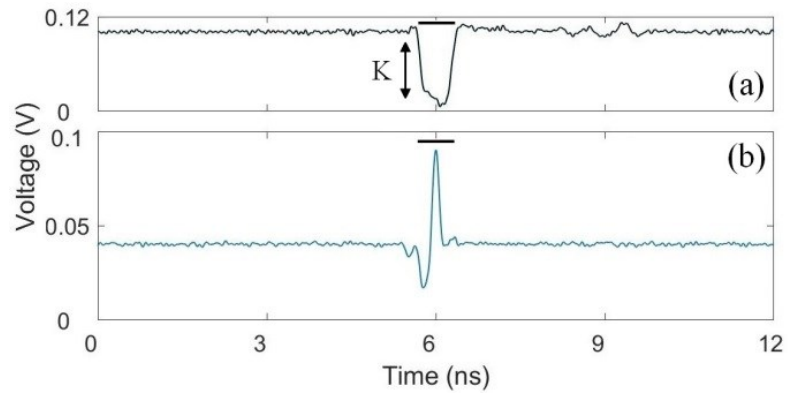

Fig. 3. (a, in black) Measured time series of the input signal with encoded negative perturbations (stimuli) optically-injected into a VCSEL-neuron operating at the wavelength of $1300 \mathrm{~nm}$. (b, in blue) Time series measured at the output of the $1300 \mathrm{~nm}$ VCSEL-Neuron showing the firing of a sub-ns spike in response to the incoming perturbation. The perturbation (stimulus) had a temporal duration $\left(\mathrm{t}_{\mathrm{d}}\right)$ equal to $0.64 \mathrm{~ns}$ and an strength of $\mathrm{K}=0.806$. The VCSEL device was driven with an applied bias current of $2.20 \mathrm{~mA}$ at the temperature of $300 \mathrm{~K}$. The constant injection power level was set equal to 270 $\mu \mathrm{W}$ and the initial frequency detuning between the injected signal and the VCSEL's resonance was equal to $\Delta \mathrm{f}=-10.3 \mathrm{GHz}$.

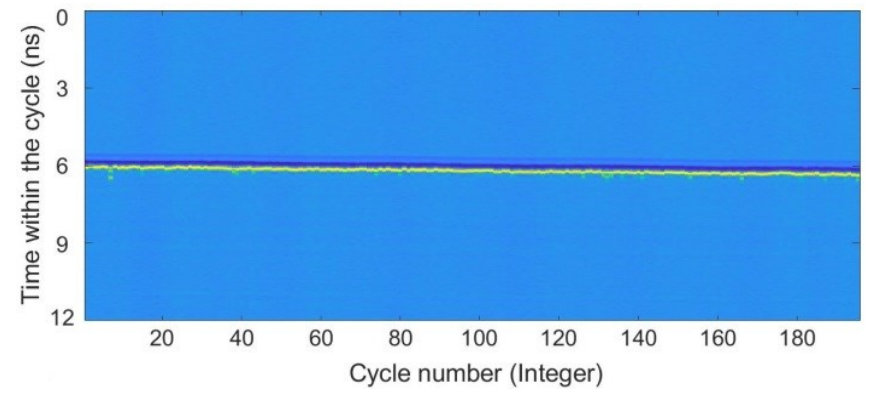

Fig. 4. Temporal map obtained from measured time-series at the output of the $1300 \mathrm{~nm}$ VCSEL-Neuron for the experimental conditions used in Fig. 3. The map merges the response of the VCSEL-Neuron to 200 consequetive incoming perturbations in one plot with each of them firing an identical spike in the device. Peaks and troughs of intensity are represented by yellow and dark blue respectively.

Fig. 3(a) plots a typically injected optical signal from the tunable laser characterized by a constant optical power level with sudden perturbations in the form of short 'power drops' (indicated by solid black lines in Fig. 3) with time-duration $t_{d}$ and strength $\mathrm{K}$, parameters than can be fully tuned. In particular, Fig. 3(a) illustrates the case of an optically injected signal with an incorporated perturbation (stimulus) of $0.64 \mathrm{~ns}$ whilst Fig. 3(b) depicts the response of a VCSEL-neuron (operating at $1300 \mathrm{~nm}$ ) to this injected signal. As seen in Fig. 3(b) prior to the arrival of the perturbation the VCSEL-Neuron exhibits a constant optical output level as it is injection-locked to the externally injected signal. The arrival of the perturbation breaks this equilibrium, unlocks the device from the external light injection, triggering as a result a spiking event. The latter had a temporal duration of $\sim 100 \mathrm{ps}$, multiple orders of magnitude faster than the millisecond timescales of biological neurons. This response is in fact analogous to the so-called phasic spiking dynamics in neurons where a single spike is fired upon the arrival of a stimulus into the cell [58]. Fig. 4 shows a temporal map which merges in a single plot the response of the VCSEL-Neuron to 200 consecutive arriving perturbations for the same conditions used in Fig. 3. A folding parameter equal to the period between consecutive perturbations was used to create the map in Fig. 4 [37]. The color code in Fig. 4 is chosen such that dark blue and yellow 
colors depict respectively low and high intensity levels. The map in Fig. 4 clearly demonstrates the reproducibility of the activated spiking response. This can be seen from the continuous yellow line in the map, which plots the intensity maxima of the 200 identical spikes obtained at the VCSELNeuron's output following the arrival of 200 consecutive perturbations.

Moreover, by simply controlling the temporal length of the incoming perturbations, it is possible to fully tune the number of spikes fired by a VCSEL-Neuron. Fig. 5 shows the results achieved when a VCSEL-Neuron is subject to perturbations with different temporal lengths equal to $2.06 \mathrm{~ns}, 2.91 \mathrm{~ns}$ and 4.52 ns respectively. Fig. 5 shows that different spike regimes with increasing number of spikes from 1 to 3 (for this particular case of analysis) are obtained at the output of the VCSEL-Neuron as the temporal length of the perturbation is grown. This type of response is indeed analogous to the socalled tonic spiking dynamics in biological neurons that fires spikes continuously for the duration of a stimulus [58]. In addition to these neuronal dynamical regimes our results showed that VCSEL-Neurons can also emulate at ultrafast speeds other neuronal responses including thresholding for spike firing, spike latency and spike rate encoding, showing consistency with the findings of the initial report [37].

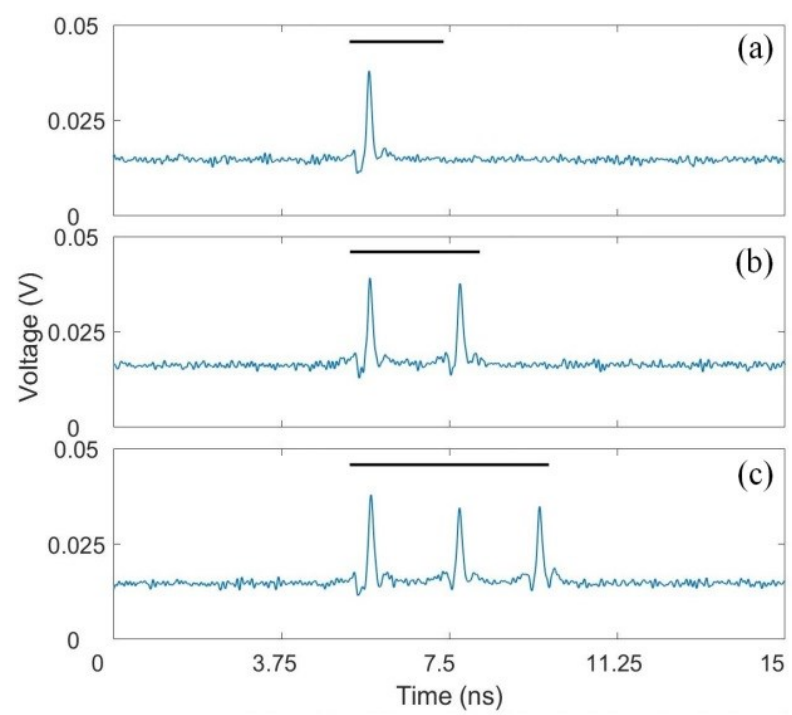

Fig. 5. Time series measured at the output of a VCSEL-Neuron (operating at $1310 \mathrm{~nm}$ ) subject to incoming optically-encoded stimuli (marked by solid black lines) of increasing temporal duration, $t_{d}$ of (a) $2.06 \mathrm{~ns}$ (b) $2.91 \mathrm{~ns}$ and (c) $4.52 \mathrm{~ns}$ and constant stimulation strength of $\mathrm{K}=0.1$. The VCSEL-Neuron was driven with a current of $3.6 \mathrm{~mA}$ at $298 \mathrm{~K}$. An injection power of $237 \mu \mathrm{W}$ (with added perturbations) and an initial frequency detuning of $\Delta f=-5.65$ $\mathrm{GHz}$ were set between the tunable laser and the VCSEL-Neuron.

We have also studied the activation of sub-ns spiking regimes in VCSEL-neurons subject to electronically encoded perturbations (see previous report [38] for full details). Fig. 6 depicts the setup used for this work. This is similar to that in Fig. 2, as it also uses an optically-injected VCSEL subject to external perturbations (stimuli). However, now the latter (in the form of short 'power raises') are electronically-encoded into the bias current applied to the VCSEL-Neuron. The optical injection conditions, including the injection strength and initial frequency detuning, are set so that the device is initially injection-locked to the external optical signal therefore producing a constant optical power output. Then the arrival of a perturbation breaks this equilibrium making the VCSEL-Neuron briefly unlock the external optical signal triggering the firing of a spiking optical output. This response is shown in Fig. 7, where experimental time-series, measured at the output of a VCSEL-Neuron (operating at $1310 \mathrm{~nm}$ ) under the electronic-injection of perturbations (stimuli) with different temporal durations, are plotted. Fig. 7 reveals that sub-ns spiking patterns, with increasing number of spikes (from 1 to 4), are obtained from the VCSEL-Neuron as the temporal length of the stimuli (marked by solid black lines in Fig. 7) are lengthened from $1.07 \mathrm{~ns}$ to $2.35 \mathrm{~ns}$.

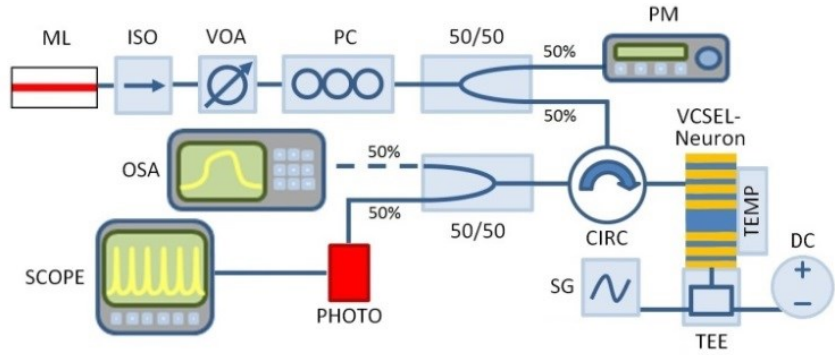

Fig. 6. Setup used for the electronically-controlled activation of sub-ns spiking regimes in a VCSEL-neuron subject to external optical injection. ML-master laser, ISO-optical isolator, VOA-variable optical attenuator, PC-polarization controller, PM-power meter, CIRC-circulator, TEMP-temperature controls, SG-electrical signal generator, TEE-bias tee, DC-laser driver, PHOTOphotodetector, SCOPE-oscilloscope, OSA-optical spectrum analyser. (See [38] for full details).

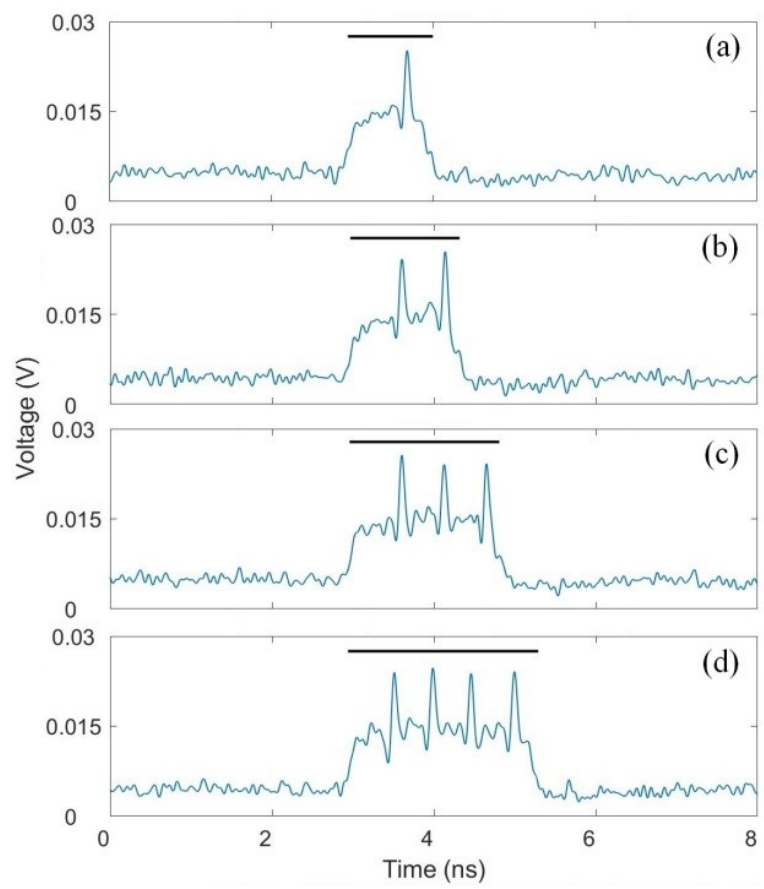

Fig. 7. Measured time series at the output of a VCSEL-Neuron with operation at $1310 \mathrm{~nm}$ subject to electronic perturbations with different controlled temporal duration $\mathrm{t}_{\mathrm{d}}$ of (a) $1.07 \mathrm{~ns}$ (b) $1.34 \mathrm{~ns}$, (c) $1.90 \mathrm{~ns}$ and (d) $2.35 \mathrm{~ns}$, respectively. The VCSEL-Neuron was driven with a current of $6.0 \mathrm{~mA}$ and the electronically-encoded stimuli increased the bias by $3.72 \mathrm{~mA}$ (at $293 \mathrm{~K}$ ). A constant optical injection power of $252 \mu \mathrm{W}$ at an initial frequency detuning of $\Delta \mathrm{f}=-5.65 \mathrm{GHz}$ between the ML and the VCSEL-Neuron was used to injection lock the solitary laser output. (See [38] for full details). 
The results included in Fig. 1-7 therefore pave the way towards optically- and electronically-controlled artificial excitatory VCSEL-based optical neurons towards future implementations of neuromorphic laser neuron networks. We must also note that although a full investigation on the ultimate speed limits of our approach for spiking VCSELNeurons (in terms of the measured refractory times) is beyond the scope of the current manuscript; nevertheless, our experimental findings (including results for previously reported [37-40] and ongoing activities) reveal sub-ns refractory times between consecutively fired spikes ranging from $\sim 500 \mathrm{ps}$ (and lower values) well below the $1 \mathrm{~ns}$ benchmark [37-40] to a few ns depending on the initially set system conditions. In our opinion the operation speed of the VCSEL-Neuron is ultimately governed by the fast dynamics of the device when biased above threshold (due to the stimulated emission of photon dynamics). The speed will be therefore limited by the device's relaxation oscillation frequency which for the commercially-available devices of this work is in the order of a few $\mathrm{GHz}$ (see [51] for details); hence enabling sub-ns speed operation rates without the need for any specific device design optimization stage.

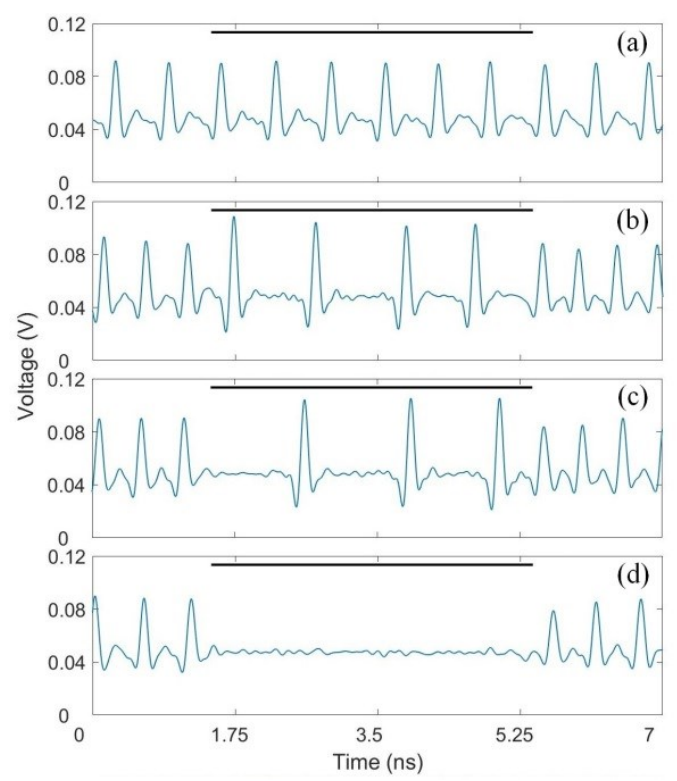

Fig. 8. Measured time series showing controllable spike inhibition in a VCSEL-neuron (at $1300 \mathrm{~nm}$ ) subject to optically-injected signals with encoded perturbations (marked by solid black lines) with increasing strength, $\mathrm{K}$ of (a) 0 (b) 0.219 , (c) 0.516 and (d) 0.803 and constant $\mathrm{t}_{\mathrm{d}}=7.45$ ns. An injection power level of $35.8 \mu \mathrm{W}$ and a frequency detuning of $\Delta \mathrm{f}=-2.77 \mathrm{GHz}$ was set between ML and VCSEL-Neuron. The latter was driven with a current of $2 \mathrm{~mA}$ at $293 \mathrm{~K}$. (See [39] for full details).

\section{B. Controlled Spiking Inhibition in VCSEL-Neurons}

Recently, we have also demonstrated that VCSEL-Neurons can controllably suppress a pre-existing spiking output; hence behaving also as inhibitory neurons (see previous report [39] for full details). In this work we have also used the experimental setup in Fig. 2. The only change is that the external perturbations are changed here to short 'power raises' (with controlled temporal length and strength) instead of the 'power drops' used to obtain the results in Figs. 3-5. Fig. 8 plots measured time traces when perturbations (marked by solid black lines in Fig. 8) with increasing strength from $\mathrm{K}=0$ to $\mathrm{K}=0.803$ and constant temporal length of $t_{d}=7.45 \mathrm{~ns}$ are injected into the VCSEL-Neuron. Here, the mean level of the injected signal is set so it triggers a continuous spiking response at the device's output. In this situation, the spiking activity can be inhibited under the arrival of perturbations, providing these exceed an intensity threshold upon which the VCSEL injection-locks to the external signal (yielding therefore a constant output). This is demonstrated in Fig. 8 where it is shown that the system's output evolves from a constant (sub-ns) spike firing output (Fig. 8(a)) to a full suppression of the spiking activity upon the arrival of a strong enough perturbation (Fig. 8(d)). The plots in Fig. 8 reveal that initially, when no perturbation enters the VCSEL-Neuron (Fig. 8(a)), a constant spiking output is obtained. Then, as the strength of the incoming perturbation is grown (see Figs. 8(bd)), the inter-spiking interval increases until the spiking response is fully suppressed for a strong enough perturbation (Fig. 8(d)). Full details on this work can be found in [39].

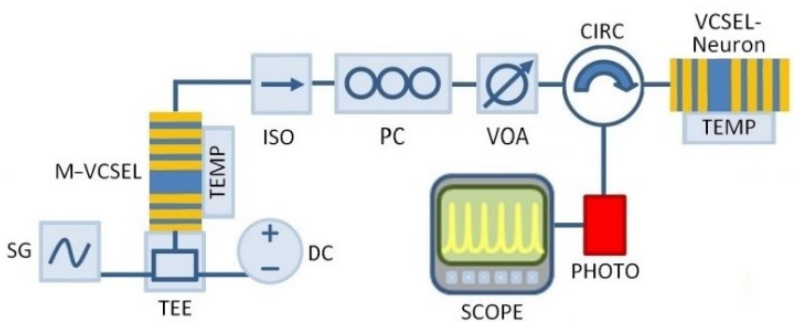

Fig. 9. Experimental setup used to controllably inhibit spiking dynamics in a synapsed VCSEL-Neuron connected to a master M-VCSEL subject to electronically encoded perturbations. Here, $1550 \mathrm{~nm}$-VCSELs were used. TEE-bias tee, SG-signal generator, DC-power supply, ISO-optical isolator, VOA-variable optical attenuator, PC-polarization controller, CIRC-circulator, TEMP-temperature controls, PHOTO-photodetector, SCOPE-oscilloscope.

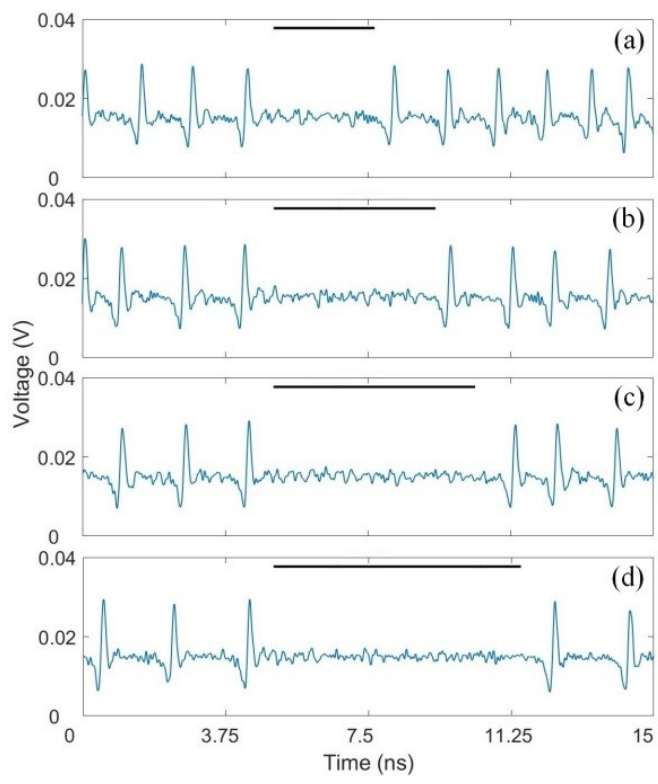

Fig. 10. Time series revealing controlled spike inhibition in a VCSEL-Neuron under external optical injection from a Master VCSEL subject to electricallyencoded perturbations with temporal duration $t_{d}$ of (a) $2.66 \mathrm{~ns}$ (b) $4.29 \mathrm{~ns}$, (c) $5.35 \mathrm{~ns}$ and (d) $6.61 \mathrm{~ns}$, respectively. A bias current of $4.16 \mathrm{~mA}$ was applied to the Master VCSEL and the electrically-encoded perturbations increased the bias by $3.2 \mathrm{~mA}$. A constant injection power of $42 \mu \mathrm{W}$ and a frequency detuning of $\Delta \mathrm{f}=-2.83 \mathrm{GHz}$ were set between the Master VCSEL and the VCSEL-Neuron. The latter was driven with a bias current of $4.06 \mathrm{~mA}$ at 294 $\mathrm{K}$. Both VCSELs used in this work operated at the wavelenght of $1530 \mathrm{~nm}$. 


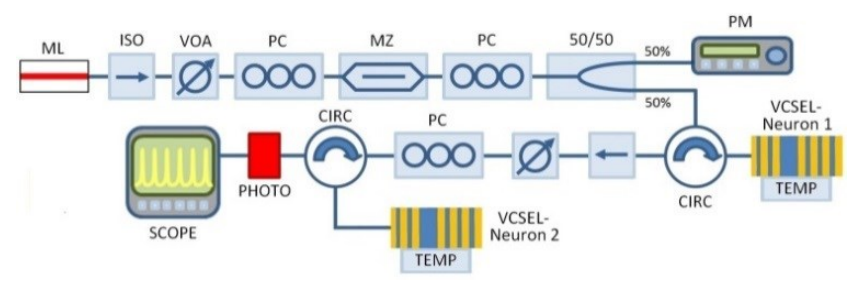

Fig. 11. Setup used to study the communication of spiking signals from a first VCSEL-Neuron (VCSEL-Neuron 1) to another synapsed VCSEL-Neuron (VCSEL-Neuron 2) in the system. Devices operating at $1310 \mathrm{~nm}$ were used in this work. ML-master laser, ISO-optical isolator, VOA-variable optical attenuator, PC-polarization controller, MZ-mach-zehnder amplitude modulator, PM-power meter, CIRC-circulator, TEMP-temperature controls, PHOTO-photodetector, SCOPE-oscilloscope.
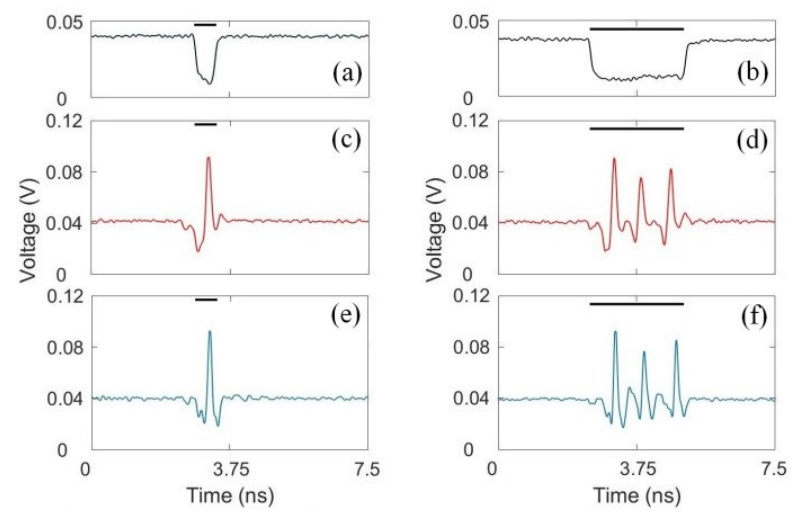

Fig. 12. (a-b, in black) Time series of the input signals encoded with perturbations of durations $t_{d}=0.61 \mathrm{~ns}(\mathrm{a})$ and (b) $2.74 \mathrm{~ns}$ injected into the VCSEL-Neuron 1. (c-d, in red) Time series at the output of the VCSELNeuron 1 and (e-f, in blue) at the output of (synasped) VCSEL-Neuron 2. VCSELs operating at $1300 \mathrm{~nm}$ were used in these experiments. Signals injected from the tuneable laser into VCSEL-Neuron 1 were set with an initial frequency detuning of $\Delta \mathrm{f}=-3.55 \mathrm{GHz}$ and an constant power of $102 \mu \mathrm{W}$. The latter was driven with a current of $1.5 \mathrm{~mA}$ at $298 \mathrm{~K}$. An injection power of $56.2 \mu \mathrm{W}$ and a frequency detuning of $\Delta \mathrm{f}=-3.55 \mathrm{GHz}$ were set for the injection between the VCSEL-Neurons 1 and 2. The latter was driven with a current of $1.35 \mathrm{~mA}$ at $292 \mathrm{~K}$.

Very recently, we have also investigated and produced new results on the achievement of spiking inhibition responses in VCSEL-Neurons under electronically encoded perturbations. Fig. 9 shows the experimental setup used for this study, consisting of two unidirectionally coupled VCSELs operating at $1530 \mathrm{~nm}$. Here, electronically-encoded perturbations (short temporal 'power raises') are added to the applied bias current of the (Master) M-VCSEL whose output is optically-injected into the VCSEL-Neuron (behaving as an inhibitory optical neuron). The injection conditions, namely injection strength and initial frequency detuning, are set so that initially the VCSEL-Neuron is continuously firing sub-ns long spiking signals (as the injection drives the VCSEL-Neuron close to but outside the injection-locking range). Then, the arrival of a perturbation (short power raises electronically-added in the bias current of the M-VCSEL), breaks this condition making the VCSEL-Neuron briefly cross into the injection locking region and as a result produce a constant power output. This is demonstrated in Fig. 10, which shows that the spiking output of the VCSEL-Neuron (at $1530 \mathrm{~nm}$ ) is controllably inhibited upon the arrival of different (electronically-encoded) perturbations with growing temporal durations from $2.66 \mathrm{~ns}$ to $5.35 \mathrm{~ns}$.
The unreported results in Fig. 8-10 therefore open the door to both optically- and electronically-controlled inhibitory artificial optical neurons with VCSELs for future use in neuromorphic photonic network implementations.

\section{Spiking communication between VCSEL-Neurons}

For the future development of neuromorphic networks of optical neurons the successful transmission of diverse photonic spiking regimes at ultrafast speeds between interconnected neurons in a circuit/network is key. We continue to focus on this important aspect and have previously analyzed the communication of spiking signals between coupled VCSEL-Neurons [40]. Specifically, using the experimental system of Fig. 11 we investigated the propagation of spiking dynamical signals at sub-ns rates between two unidirectionally coupled VCSEL-Neurons. Here, in a similar demonstration, external optical signals with encoded perturbations are generated by externally modulating the output of a tunable laser before they are injected into the VCSEL-Neuron 1 in the setup of Fig. 11. VCSEL-Neuron 1 responds by firing a controllable spiking response (as in Figs. 3-5) that is in turn propagated to the VCSEL-Neuron 2. The outputs of both devices are analyzed with a fast real time oscilloscope and an OSA to observe the communication of the spiking patterns between them. Full details about this work and the system in Fig. 11 can be found in [40].

Fig. 12 plots experimental results studying the communication of excitatory spiking signals between two VCSEL-Neurons operating at $1300 \mathrm{~nm}$. The two columns in Fig. 12 plot results obtained when two perturbations with different temporal lengths, $0.61 \mathrm{~ns}$ (left column, Fig. 12(a,c,e)) and 2.74 ns (right column, Fig. 12(b,d,f)), are injected into VCSEL-Neuron 1. In both cases spiking patterns with different number of spikes: 1 spike for $t_{d}=0.61 \mathrm{~ns}$ and 3 spikes for $t_{d}=2.74 \mathrm{~ns}$ are obtained at the output of VCSELNeuron 1, as seen in the time series included in the red plots in Fig. 12(c-d). The responses from VCSEL-Neuron 1 are communicated to VCSEL-Neuron 2 whose outputs are shown in the blue plots in Fig. 12(e-f). In both cases studied, the results obtained at the output of the system reveal that the same spiking pattern, with similar number of spikes, spike features and amplitudes is also obtained from VCSEL-Neuron 2. Therefore, successful communication of excitatory spiking dynamical signals at sub-ns speed rates between two interconnected VCSEL-Neurons is demonstrated. The maps included in Fig. 13 merge the responses obtained from VCSEL-Neuron 1 (Fig. 13(a)) and VCSEL-Neuron 2 (Fig. 13(b)) when 100 consecutive equal perturbations (with $t_{d}=$ $0.61 \mathrm{~ns}$ ) are injected into the first device. As expected, a single spike is fired by VCSEL-Neuron 1 for each and all of the 100 incoming perturbations (as revealed by the straight line in the map in Fig. 13(a). In Fig. 13(b) the map demonstrates that every single one of the 100 spikes obtained from VCSELNeuron 1 are successfully communicated to VCSEL-Neuron 2. Furthermore, we have also recently demonstrated that this scheme also permits the successful communication of inhibitory spiking signals at sub-ns rates between two 
VCSEL-Neurons [41]. These works [40,41] therefore pave the way to larger interconnected systems/networks of VCSELNeurons for future neuromorphic photonic systems for computing and Artificial Intelligence. This is the subject of our ongoing research, including the investigation of different network interconnectivity architectures between multiple artificial VCSEL-Neurons. (a)

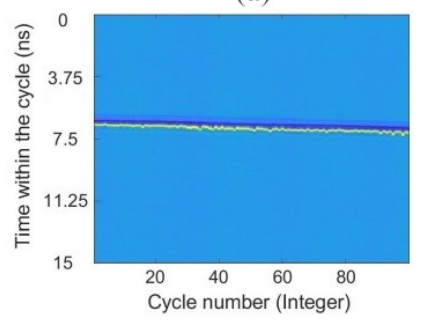

(b)

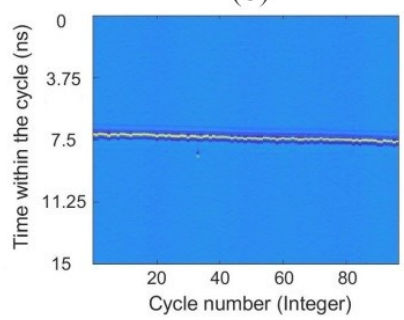

Fig. 13. Temporal maps measured at the outputs of (a) VCSEL-Neuron 1 and (b) (synapsed) VCSEL-Neuron 2. 100 consectuitve perturbations of $t_{d}=0.61$ ns are injected into VCSEL-Neuron 1 which reacts by firing a single spike for all of them. These are transmitted to VCSEL-Neuron 2 which reproduces the same spiking pattern.

\section{NeUromorphic Photonic SPIKING Processing TASKS WITH VCSEL-NEURONS}

\section{A. Spiking photonic memory with a single VCSEL-Neuron}

Our attention has also recently turned to the development of neuromorphic photonic systems with VCSEL-Neurons able to perform spiking processing tasks. In this new work, we have used VCSELs operating at telecom wavelengths (1310 and $1550 \mathrm{~nm}$ ), fully compatible with current optical networks and data centre technologies; hence offering great potentials for novel routes towards functional brain-inspired photonic systems. Initially, we have focused on the development of photonic spiking memory modules operating at sub-ns speed rates using VCSEL-Neurons as building blocks. The first configuration we have used is the so-called autaptic neuronal architecture [11]. Here, the output of a VCSEL-Neuron is fed back into itself via a delayed feedback loop. The delayed feedback loop enables the storage of controllable photonic spiking patterns, with the duration of the feedback loop determining the length of the buffer memory.

Fig. 14 plots the setup built to experimentally investigate this autaptic spiking VCSEL-Neuron system. Optical signals with encoded perturbations (short 'power drops', as in Fig. 3) are generated from an externally-modulated tunable laser. These are injected into the VCSEL-Neuron via an optical circulator. Polarization controllers and a variable optical attenuator are included to control the polarization and injection strength of the externally-injected optical signals. The output of the VCSEL-Neuron is divided into two branches by means of a $1 \times 2(50 / 50)$ coupler. The first optical paths is connected to a fast $(>9.5 \mathrm{GHz})$ photodiode and a fast $(13$ $\mathrm{GHz}$ ) real time oscilloscope for analysis. The second optical branch is used to generate the delayed feedback loop. This includes an optical isolator to avoid reflections, and an extra variable optical attenuator and polarization controller, to control the injection strength and the polarization of the signals fed back into the VCSEL-Neuron. The latter is connected to the second input port of the $2 \times 2$ (50/50) coupler which closes the delayed feedback loop.

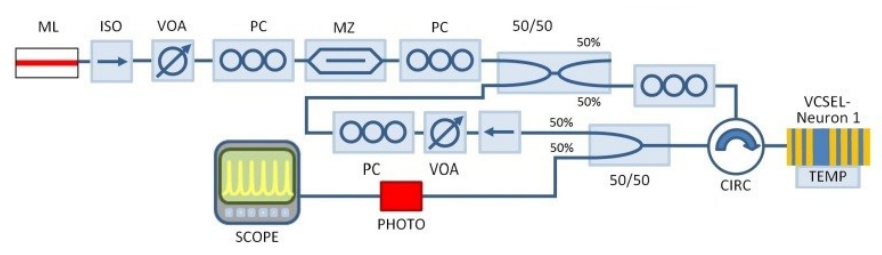

Fig. 14. Setup used to build a photonic spiking memory with an (autaptic) VCSEL-Neuron under optical feedback. ML-master laser, ISO-isolator, VOAvariable optical attenuator, PC-polarization controller, CIRC-circulator, TEMP-temperature controls, PHOTO-photodetector, SCOPE-oscilloscope.

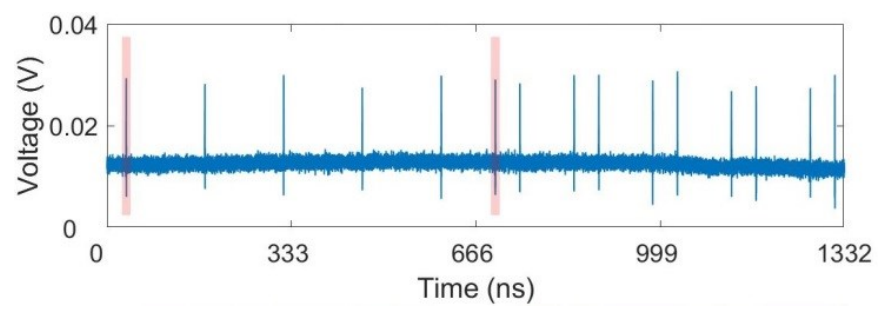

Fig. 15. Time traces obtained from a VCSEL-Neuron subject to self-feedback (autaptic configuration) and to optical injection of signals with encoded stimuli. The optical injection triggers the firing of spikes (highlighted in red) which are fed-back into the VCSEL-Neuron via a 142 ns-long delay loop following which the spiking pattern is regenerated. 9 cycles totaling a memory time in excees of $1.3 \mu \mathrm{s}$ are plotted. The VCSEL-neuron operated at $1530 \mathrm{~nm}$ and was driven with a current of $5.30 \mathrm{~mA}$ at $293 \mathrm{~K}$. An initial frequency detuning of $\Delta \mathrm{f}=-2.53 \mathrm{GHz}$ and constant injection and feedback optical powers of $29 \mu \mathrm{W}$ and $10.4 \mu \mathrm{W}$ were set.

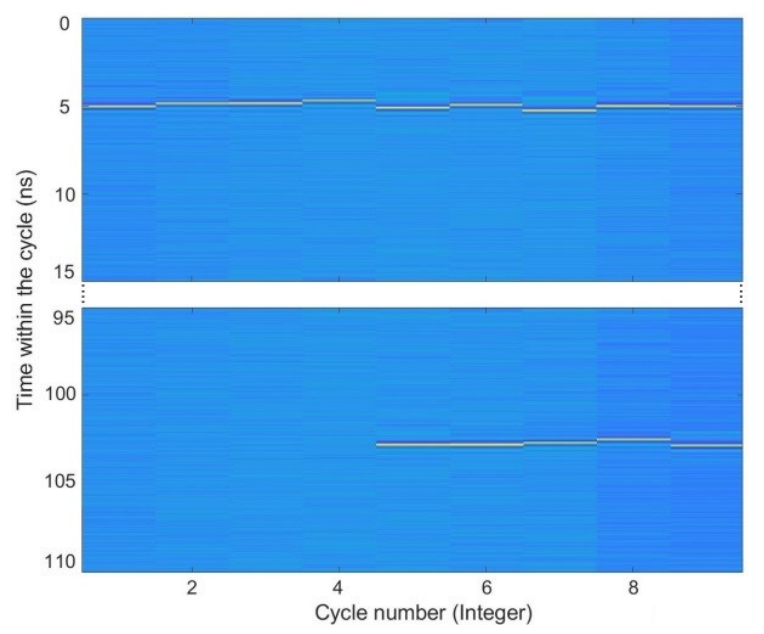

Fig. 16. Temporal map of the VCSEL-Neuron in the self-feedback configuration shown in the setup of Fig. 14. The map plots the consistency of the stored spiking responses which are regenerated every $142 \mathrm{~ns}$ (delay loop time). The initial stimuli-induced spiking response is stored for 9 full cycles whilst the second activated spike is triggered after the $4^{\text {th }}$ cycle and kept stored thereafter.

Fig. 15 plots experimental results obtained with the developed autaptic VCSEL-Neuron spiking memory system. The highlighting red boxes in Fig. 15 indicate the time instants at which external perturbations enter the VCSEL-Neuron, triggering the firing of a spike. This is fed back into the VCSEL-Neuron via a delayed loop with a set time length of $\tau$ $=142 \mathrm{~ns}$, following which the device fires an analogous spike. Fig. 15 shows therefore that the externally-generated spike is regenerated every $\tau=142 \mathrm{~ns}$ (spikes are fired at time instants separated by $142,284,426,568$ ns from the first induced 
spike). The first half of the time series in Fig. 15 therefore shows that a 1-spike pattern stored in the autaptic VCSELNeuron memory system. This situation is kept until a new perturbation arrives into the VCSEL-Neuron at a time instant of $\sim 675 \mathrm{~ns}$. This new perturbation triggers the firing of a second spike which is also stored in the system. The second half of the time series in Fig. 15 shows that after $\sim 675$ ns, the pattern stored changes from a 1-spike to a 2 -spike pattern which is regenerated every $\tau=142 \mathrm{~ns}$. This is also illustrated graphically in Fig. 16, plotting two 15 ns-long sections (from 0 to $15 \mathrm{~ns}$ and from 95 to $110 \mathrm{~ns}$ ) of the total delayed feedback loop time $\tau=142 \mathrm{~ns}$ (used as the folding parameter to create the map). Fig. 16 reveals that at first a 1-spike pattern is stored in the VCSEL-Neuron, as a single spike appears at the same time instant ( $\sim 5 \mathrm{~ns})$ in the cycle, as seen in the upper section of the map. Then after 4 cycles, the second perturbation arrives, changing the stored signal to a 2 -spike pattern with the second spike appearing at equal time instants ( 103 ns) in the cycle, as seen in the lower section of the map.

We must note that in our experiments the external perturbations (short pulses) were generated with a signal source operating with a repetition frequency of $15 \mathrm{MHz}$; hence a new external perturbation enters the autaptic VCSELNeuron every $666 \mathrm{~ns}$. As a result, the spiking pattern stored in the system changes continuously after a few cycles, as seen in Figs. 15 and 16. For clarity Fig. 15 plots a time trace showing the system's response during 9 consecutive cycles to demonstrate the storage and regeneration of different controllable spiking patterns. Although a full investigation of the operation limits of the reported autaptic VCSEL-Neuron spiking memory is beyond the scope of this work, we can nevertheless discuss a few important aspects regarding system's performance. Firstly, the system will be able to keep the regenerated spiking patterns stable in the system for higher number of cycles (much larger than the duration of delayed feedback loop). Secondly, the system will also be able to store spike patterns with higher number of spikes $(>2)$ per cycle. Additionally, the results in Figs. 15 and 16 already demonstrate that a spike event can be 'written' and stored in the autaptic VCSEL-Neuron memory. However, other interesting possibilities worth of future investigation are to determine the system's ability to selectively 'erase' stored spikes as well as to state the minimum temporal separation between regenerated spikes (ultimately limiting the total capacity of the spiking memory). Regarding the latter, we believe that the maximum system's capacity (highest quantity of stored spikes per cycle) would be ultimately limited by the refractory time of the spiking VCSEL-Neuron and the length of the delayed feedback loop (with delay proportional to capacity). Further experimental and numerical investigations, beyond the scope of this work, will be needed to identify the ultimate limitations of the spiking memory system to determine its maximum performance.

\section{B. Spiking photonic memory with coupled VCSEL-Neurons}

We have also started to explore the potentials of interconnected VCSEL-Neurons for spiking memory modules at sub-ns rates. In particular, we have built a system with two VCSEL-Neurons connected in a mutual-coupling architecture [27][59] (see setup in Fig. 17). This includes two VCSELs operating at $1550 \mathrm{~nm}$, which are mutually-coupled via a 217 ns-long delay coupling loop. The latter is built using two $1 \times 2(50 / 50)$ couplers and two circulators to allow mutual reinjection of the VCSEL-Neurons' outputs. These were collected from the spare $1 \times 2$ couplers' ports and send for analysis with a real-time oscilloscope and an OSA. Moreover, the two branches forming the coupling loop incorporated a variable optical attenuator and a polarization controller to respectively control the polarization and strength of the injected signals into the VCSEL-Neurons. The $1550 \mathrm{~nm}$ VCSELs used here had similar emission wavelengths so were matched by tuning the devices' bias currents and operating temperatures. The system was designed for the two VCSELNeurons to be injection-locked to each other; hence initially yielding constant outputs. Nonetheless, the system was purposely driven close to the boundaries between the locking/unlocking regions so that noise could force it to transit between those two dynamical regimes, triggering as a result the firing of self-generated spike patterns. These are reinjected alternatively into both VCSEL-Neurons (via the delayed coupling loop), enabling their storage in the system.

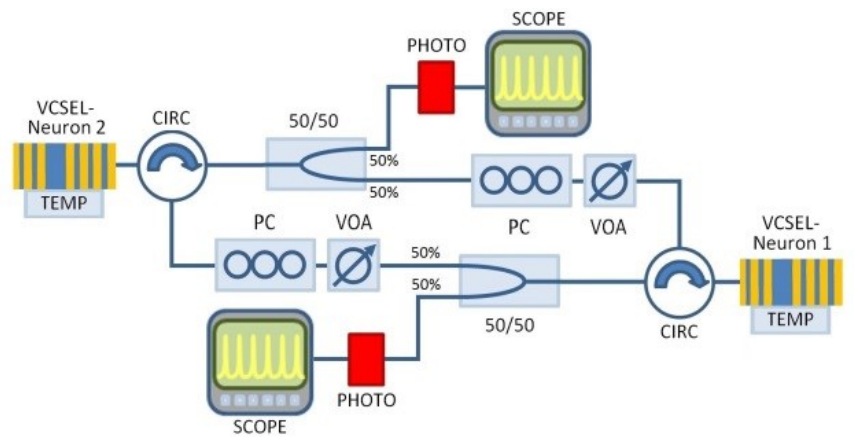

Fig. 17. Setup used to develop a neuromorphic photonic spiking memory system with mutually coupled VCSEL-Neurons. VOA-variable optical attenuator, PC-polarization controller, CIRC-circulator, TEMP-temperature controls, PHOTO-photodetector, SCOPE-oscilloscope.

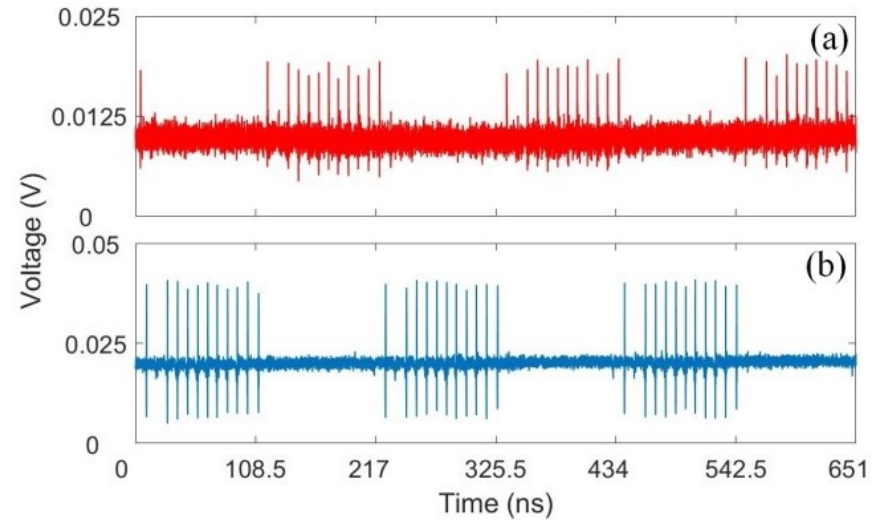

Fig. 18. Measured time-traces at the outputs of (a) VCSEL-Neuron 2 and VCSEL-Neuron 1 (b) in the setup of Fig. 17. A noise-induced spiking pattern is activated in VCSEL-Neuron 1 which is propagated and regenerated alternatively after $\tau_{\mathrm{c}} / 2=108.5 \mathrm{~ns}$ in VCSEL-Neurons 2 and 1 . Devices operating at $1530 \mathrm{~nm}$ were used here. VCSEL-Neurons 1 and 2 were driven respectively with bias currents equal to $6.17 \mathrm{~mA}$ (at $292 \mathrm{~K}$ ) and $6.05 \mathrm{~mA}$ (at $294 \mathrm{~K}$ ). A frequency detuning of $\Delta \mathrm{f}=-2.03 \mathrm{GHz}$ was set between devices. 
(a)

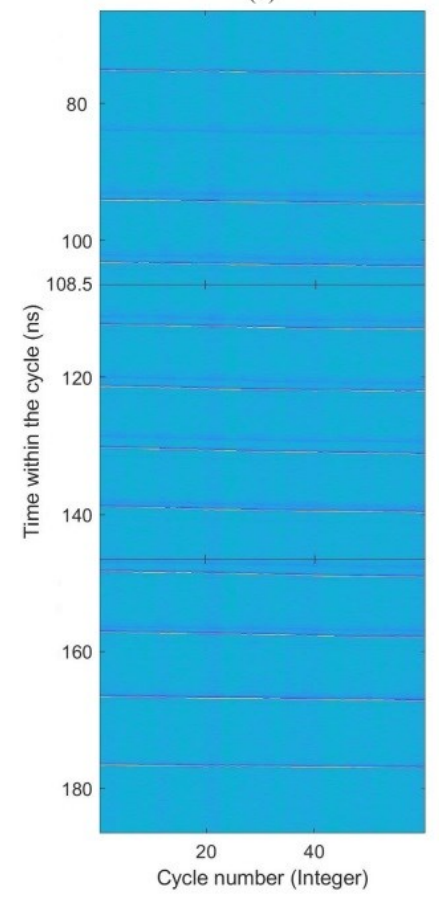

(b)

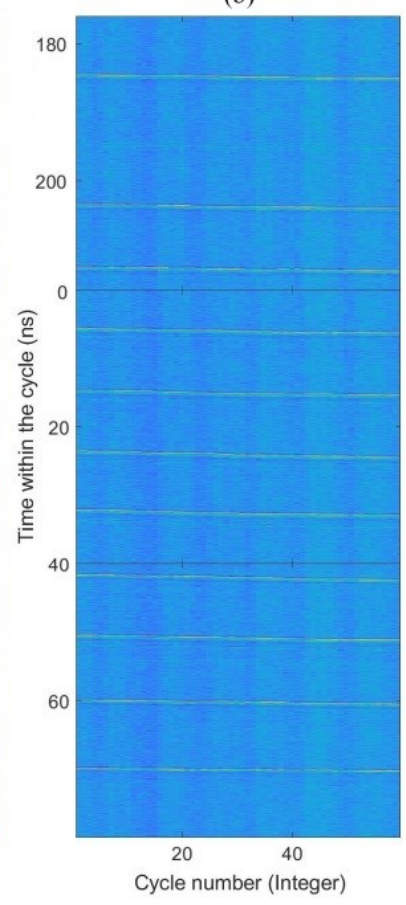

Fig. 19. Temporal map of the spiking outputs at VCSEL-Neurons 1 (a) and 2 (b). Each map plots 60 consecutive (108.5ns-long sections) of the total cycle length ( $217 \mathrm{~ns}$ coupling loop) showing the 11 spikes stored in the memory.

Fig. 18 plots measured time traces from both mutuallycoupled VCSEL-Neurons. The upper (lower) plot in red (in blue) color depicts the time series measured at the output of VCSEL-Neuron 2 (VCSEL-Neuron 1). Fig. 18 shows that noise in the system induces the self-activation of a spiking pattern in VCSEL-Neuron 1 (Fig. 18(a), in blue) characterized by a first initial sub-ns long spike followed by a 10 -spike burst (with sub-ns spike length), after a small temporal 'spike empty' gap. This self-generated spike pattern from VCSELNeuron 1 arrives into VCSEL-Neuron 2 after a time of 108.5 ns (equal to half the duration of the mutual coupling delayed loop, $\left.\tau_{\mathrm{c}}=217 \mathrm{~ns}\right)$. The second device produces the same spiking response as it regenerates the spiking pattern arriving from VCSEL-Neuron 1. After that, the output of VCSELNeuron 2 is further reinjected and once again reaches VCSELNeuron 1 after a further time of $\tau_{\mathrm{c}} / 2=108.5 \mathrm{~ns}$ (second half of the mutual coupling delayed loop), where it is regenerated again. As a result, the 11-spike pattern is stored in the coupling loop connecting both VCSEL-Neurons and it is regenerated alternatively by both devices after a temporal separation of 108.5 ns, as seen in Fig. 18.

These results are further illustrated in the temporal maps in Fig. 19. These merge data from 60 consecutive 108.5 ns-long sections (half the duration of the coupling loop length, $\tau_{c}$ ) measured at the outputs of VCSEL-Neuron 1 (Fig. 19(a)) and VCSEL-Neuron 2 (Fig. 19(b)), respectively. The maps in Fig. 19 show that the spike patterns obtained from both VCSELNeurons exhibit the same characteristics, storing the same 11spike firing pattern, with equal features, sub-ns spike duration and analogous spike separations for the 60 different cycles analyzed here, and which form a total memory time $>12 \mu \mathrm{s}$.

The only difference between the stored patterns in both devices is their respective temporal positions within the coupling loop cycle as they are shifted $108.5 \mathrm{~ns}\left(\tau_{\mathrm{c}} / 2\right)$ apart. We must note that to date, this neuromorphic photonic spiking memory system built with two mutually-coupled VCSELNeurons has been solely tested under self-generated spike patterns induced by noise in the system. We are currently upgrading our experimental arrangements to also investigate the performance of this spike memory system under controllable injection of externally-generated fast (sub-ns) perturbations, entering into either of the two interconnected VCSEL-Neurons. This is the subject of ongoing work and will be reported in due course. Additionally, although the full investigation of the storage capacity of the system (spikes per cycle) is beyond the scope of this work, in our opinion it should be possible for the proposed spiking memory architecture to store large spike patterns (going beyond the 11spike pattern reported in Figs. 18 and 19). A first simple route to achieve that result would be to investigate and test the system's capacity with longer delay lines by incorporating larger paths lengths to the feedback branches in the experimental setup depicted in Fig. 17. Future experimental and numerical studies, beyond the scope of the present work, will be needed to fully determine the system's capacity and the conditions for optimized performance.

\section{Ultrafast Emulation of Neuronal Circuits/Networks with VCSEL-based Artificial Optical Neurons}

We are also interested in neuromorphic photonic approaches for the emulation of actual neuronal circuitry as a first step towards the development of photon-enabled ultrafast spiking processing systems directly inspired by the human brain. Our first effort in this exciting research direction consists of the emulation of a biological neuronal circuit in the retina. This seemingly random decision is however a purposely choice, given the existing body of work in artificial retina systems based upon electronic circuitry approaches (see for example [60-65]) and on the readily available extensive scientific understanding of the biological neuronal circuits in the retina (see for instance [66-68]). Schematic diagrams of the retinal neuronal circuits to be emulated with neuromorphic photonic techniques based upon VCSEL-Neurons are shown in Figs. 20(a-b). These consist, in their simplest form, of two different neurons connected in series, known as Bipolar (BC) and Retinal Ganglion Cells (RCG). BCs are non-spiking neurons, yielding graded potentials of different intensities in response to inputs from the photoreceptors in the eye (receiving external light stimulation) [66-68]. The output of the BCs is connected to RGCs, which are spiking neurons converting the graded potential signals from the $\mathrm{BCs}$, into spike firing patterns for their subsequent transmission to the brain via the optic nerve for further processing [66-68]. Interestingly, there are two types of BCs and RCGs, usually referred as ON- and OFF-type BCs and RCGs, creating two different circuits in the retina producing opposite responses, ON- (see Fig. 20(a)) and OFF- (see Fig. 20(b)) circuits. The $\mathrm{ON}$ - and OFF-BC (RGC) neurons react by producing graded 
potentials (spiking signals) of opposite sign in response to the presence or absence of a light stimulus received by a photoreceptor in the eye. As a result, the ON- and OFF-type BC+RGC circuits, depicted in Figs. 20(a) and 20(b) respectively, produce different spiking outputs under the incidence of light signals or under the reduced incidence of dark conditions. A large number of such neuronal circuits are used in parallel by the retina to pre-process the incoming visual information entering the eye before further processing stages take place in the visual cortex of the brain [66-68].
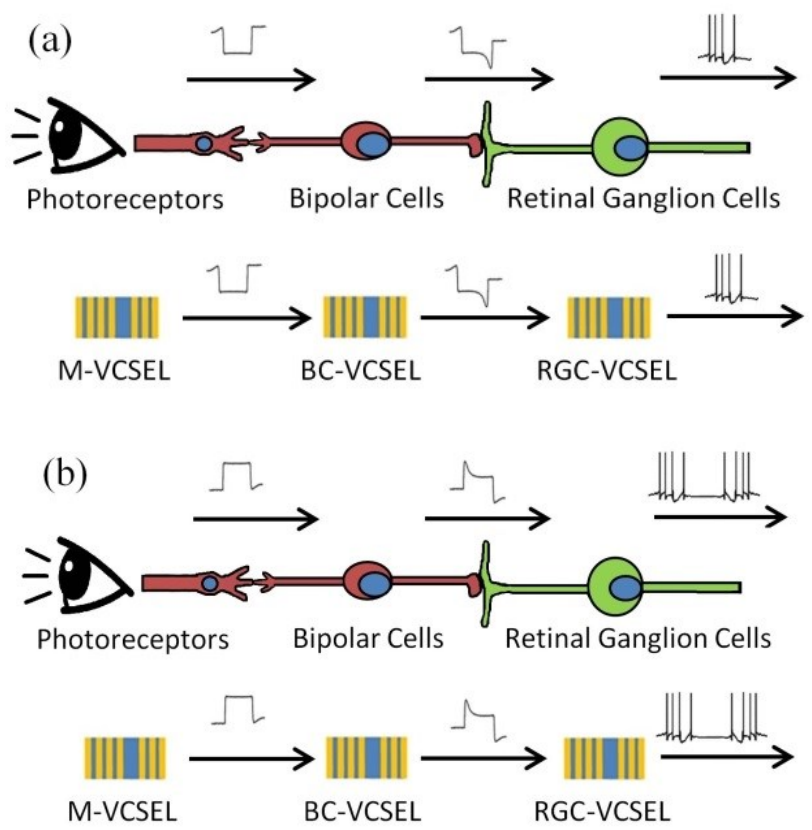

Fig. 20. (a,b) Schematics of the retinal neuronal circuits (top plots) emulated with interconnected VCSEL-Neurons (bottom plots). The circuits are formed by two types of neurons connected in series: Bipolar Cells (BC), connected to photoreceptors in the eye and Retinal Ganglion Cells (RGC) receiving inputs from BCs. These neurons are of two different types ON- and OFF-, forming two different circuits: (a) ON- (b) OFF-type BC+RGC circuits. The diagrams show the signal conversion structure in the ON- (a) and OFF- (b) type circuits in the retina performed by photoreceptors in the eye, BCs and RGCs (top plots) and the emulation of these signals using interconnected VCSELNeurons (bottom plots). M-VCSEL: Master VCSEL, BC-VCSEL: Bipolar Cell VCSEL, RGC-VCSEL: Retinal Ganglion Cell VCSEL.

We propose to emulate this basic neuronal ON-type and OFF-type BC+RGC retinal circuits using VCSEL-Neurons connected in series, as shown in the bottom plots of Fig. 20(a) and Fig. 20(b). A VCSEL-Neuron (BC-VCSEL) operates as the non-spiking $\mathrm{BC}$ neuron whilst another one (RGC-VCSEL) reproduces the response of the spiking RGC neuron. It should be noted here that in addition to providing the sub-ns spiking responses which will be needed for the optical emulation of RGCs (as reported in Section II.A in this article), VCSELs are very versatile devices also exhibiting a rich diversity of optical switching responses when subject to polarized optical injection [35,36]. Such switching responses show temporal features analogous to that of the graded potentials produced by (ON- and OFF-type) BCs. Therefore, remarkably, using the same VCSEL devices, it is possible to deliver the ultrafast emulation of the responses from both spiking and non-spiking neurons.

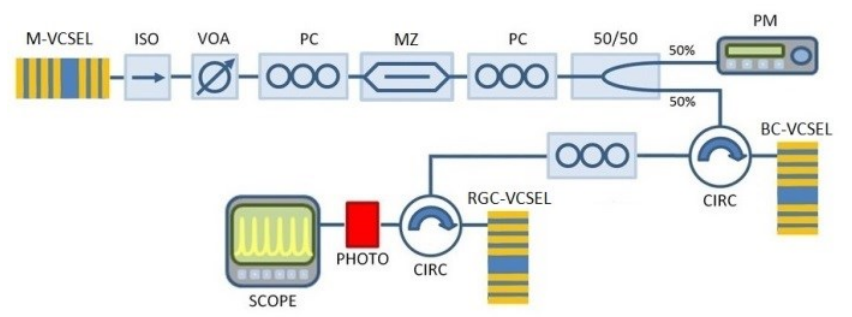

Fig. 21. Experimental setup used to emulate the operation of retinal neuronal circuits with VCSEL-Neurons. Master VCSEL (M-VCSEL), Bipolar Cell VCSEL (BC-VCSEL), Retinal Ganglion Cell VCSEL (RGC-VCSEL), ISOoptical isolator, VOA-variable optical attenuator, PC-polarization controller, MZ-Mach-Zehnder amplitude modulator, PM-power meter, CIRC-optical circulator, PHOTO-photodetector, SCOPE-oscilloscope. (a)

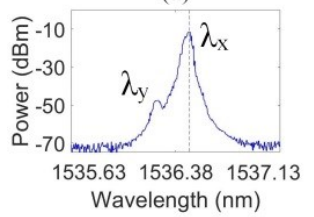

(b)

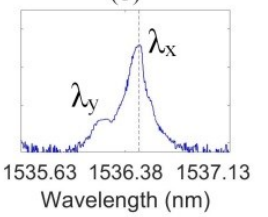

(c)

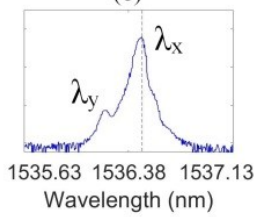

Fig. 22. Spectra of the (a) M-VCSEL, (b) BC-VCSEL and (c) RGC-VCSEL. The devices operate all at the same wavelength as the RGC-VCSEL is injection locked to BC-VCSEL which is in turn locked to M-VCSEL.

Fig. 21 plots the experimental setup developed to emulate the proposed retinal neuronal circuits using three coupled 1550nm-VCSELs. Light from a first master VCSEL (MVCSEL) is passed through an amplitude modulator (MZ) and encoded with optical stimuli in the form of short 'power drops' (ON-circuit) or 'power raises' (OFF-circuit) with controlled temporal duration $\left(\mathrm{t}_{\mathrm{d}}\right)$ and strength $(\mathrm{K})$. The intensity perturbations allow the M-VCSEL to emulate changing incident light conditions on the photoreceptors of the eye. An optical isolator (ISO) and a variable optical attenuator (VOA) are placed after M-VCSEL to prevent unwanted feedback and control the amount of power being injected into the second VCSEL emulating the operation of the $\mathrm{BC}$ in the circuit (Bipolar Cell VCSEL, BC-VCSEL). The BC-VCSEL is injection-locked to M-VCSEL's light, and remains locked to M-VCSEL's input during the intensity changes triggered by the encoded perturbations, thus creating graded potentials similar to those produced by a BC in response to different light intensity signals. Negative (Positive) amplitude graded potentials are obtained at the BC-VCSEL's output when operated as an ON- (OFF)-type BC (in response to perturbations of different sign). The output from BC-VCSEL is injected into the third VCSEL in the system (RGC-VCSEL) emulating the response of the RGC neurons. These devices are set with different operating conditions depending on the type of circuit, ON- or OFF-type, emulated. For the ON-type circuit, the RGC-VCSEL will be injection-locked to BCVCSEL's input, yielding initially a constant stable output. In that situation, the arrival of a negative graded potential from BC-VCSEL breaks the locking condition triggering the firing of a spiking pattern by RGC-VCSEL. On the other hand, in the OFF-type circuit, the conditions are set so that RGCVCSEL is close but outside the injection locking range; hence initially producing a continuous spiking output. Then, the arrival of a positive graded potential from BC-VCSEL brings the system into the injection-locking state suppressing the 

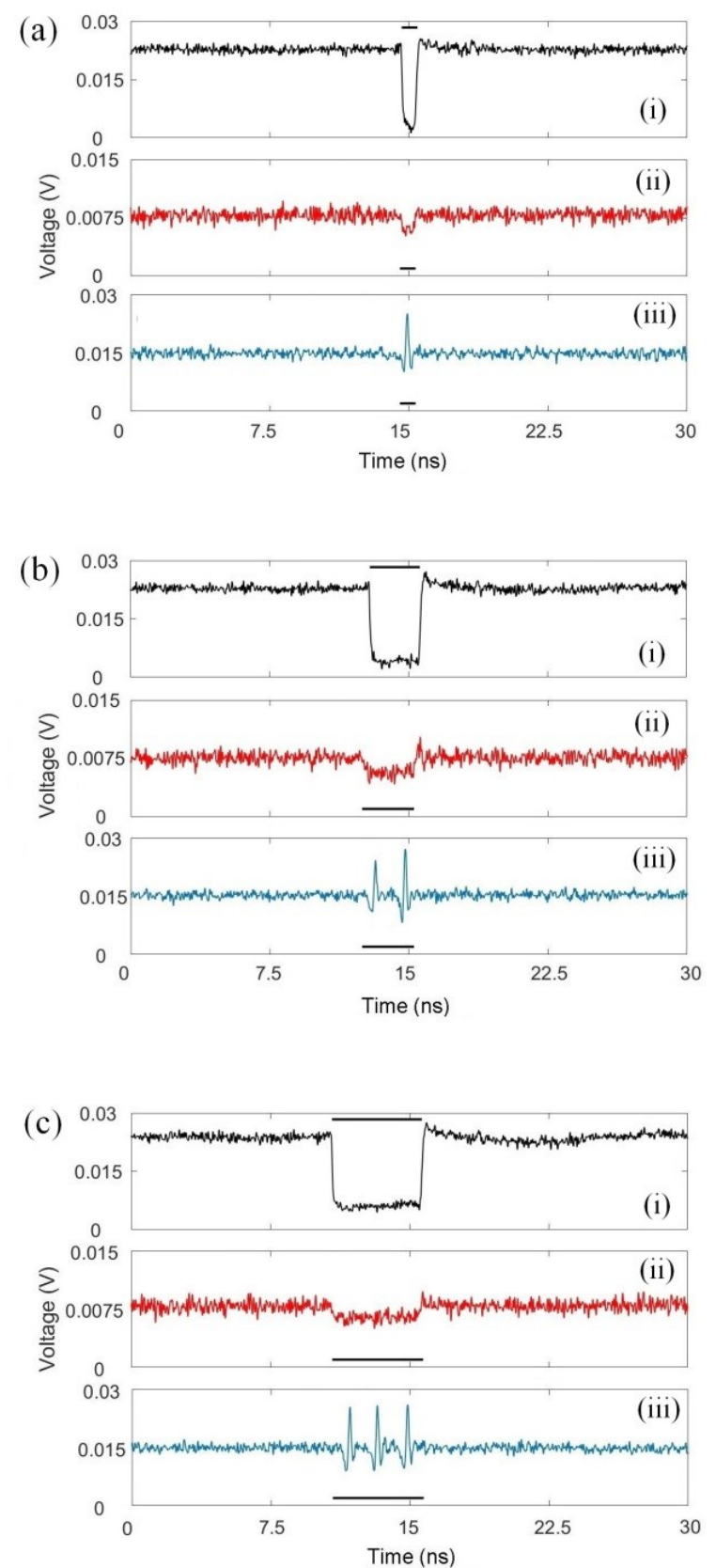

Fig. 23. Photonic emulation of an ON-type BC+RGC circuit with VCSELNeurons. Three input signals from M-VCSEL with different stimulus length of (a) $0.85 \mathrm{~ns}$, (b) $2.79 \mathrm{~ns}$ and (c) $4.90 \mathrm{~ns}$ are investigated ((i), in black). Plots (ii) (in red) depict the measured time series from BC-VCSEL. This reacts by producing graded potentials in response to the input stimuli. Plots (iii) (in blue) show the measured time series from RGC-VCSEL. This fires spiking patterns (from 1 to 3 spikes) in response to the graded potentials from BCVCSEL. VCSELs operating at $1550 \mathrm{~nm}$ were used in this work. The M-, BCand RGC-VCSELs were driven with bias currents of $4.64 \mathrm{~mA}$ (at $298 \mathrm{~K}$ ), 4.38 $\mathrm{mA}$ (at $290 \mathrm{~K}$ ) and $3.7 \mathrm{~mA}$ (at $300 \mathrm{~K}$ ) respectively to tune their emission wavelengths. The injected signals from M-VCSEL into BC-VCSEL were set with a constant power level of $70.35 \mu \mathrm{W}$ (with added $19.42 \mu \mathrm{W}$ power drops) and an initial frequency detuning of $\Delta \mathrm{f}_{\mathrm{BC}-\mathrm{M}}=-2.3 \mathrm{GHz}$. The injection from BC-VCSEL into RGC-VCSEL was set with a constant power level of 28.8 $\mu \mathrm{W}$ and a frequency detuning of $\Delta \mathrm{f}_{\mathrm{RGC}-\mathrm{BC}}=-2.53 \mathrm{GHz}$.

spiking activity (yielding a stable output instead). To analyze these responses, the outputs of all three VCSELs emulating the responses of the eye photoreceptor signals (M-VCSEL), and the outputs from the $\mathrm{BC}$ (BC-VCSEL) and RCGs (RCG-
VCSEL) are detected using fast $(>9.5 \mathrm{GHz})$ amplified photodetectors and their time-traces are analyzed with a 13 $\mathrm{GHz}$ real time oscilloscope. The wavelengths of all three VCSELs are matched by tuning their applied bias current and operating temperature. Fig. 22 plots the optical spectrum of all three VCSEL devices used in this system under typical operating conditions.

Fig. 23 plots results measured for the photonic emulation of the ON-type $\mathrm{BC}+\mathrm{RGC}$ retinal circuit. Fig. 23 shows the data obtained for three different M-VCSEL input signals, each with encoded stimuli (power drops) of different temporal duration. Fig. 23(a) shows results for when an input signal from MVCSEL (plot (i), in black) with 0.85 ns-long stimuli (or perturbations) is injected into the BC-VCSEL. Plots (ii) (in red) and (iii) (in blue) in Fig. 23(a) depict respectively the measured time series from the BC- and the RGC-VCSEL.

These show that both the BC- and RGC-VCSEL yield initially a constant output as they are injection-locked to the external injection from M-VCSEL. Then, the arrival of the stimulus (marked by solid black lines in the plots) produces a negative graded potential in the BC-VCSEL which in turn triggers the firing of a sub-ns spike by the RGC-VCSEL. Once the stimulus is removed, both the BC- and RGC-VCSELs return to their initial constant output levels. Fig. 23(b) and Fig. 23(c) depict in turn results when the input signals from M-VCSEL (i), in black) are encoded with stimulus of different temporal length, namely 2.79 ns and 4.9 ns. In both cases included, Fig. 23(b) and 23(c), the BC-VCSEL ((ii), in red) responds with a negative graded potential for the whole duration of the input stimulus, which in turn makes the RGC-VCSEL ((iii), in blue) fire spiking events for as long as the graded potentials from BC-VCSEL are present. As a result, at the system's output, the RGC-VCSEL yields either a 2-spike or a 3-spike pattern as the stimulus length from M-VCSEL are grown from 2.79 ns (Fig. 23(b)) to 4.9 ns (Fig. 23(c)). The response of the emulated ON-type BC+RGC circuit to multiple consecutive perturbations is investigated in the temporal map of Fig. 24. This merges the results obtained for the three cases investigated in Fig. 23, when 60 consecutive stimuli from MVCSEL (reproducing the output signals from photoreceptors in the eye) are injected into BC-VCSEL. The patterns obtained in the three collated maps of Fig. 24 (measured at the output of the RGC-VCSEL) clearly show that every single one of the 60 incoming stimuli trigger the firing of the same spiking pattern $(1-, 2-$, or 3-spike pattern) at the output of the ON BC+RGC circuit, for the three different cases investigated, namely 0.85 ns (Fig. 24(a)), 2.79 ns (Fig. 24(b)) and 4.90 ns (Fig. 42(c)).

Fig. 25 plots the experimental results studying the emulation of the OFF-type BC+RGC retinal circuit shown in Fig. 20. Three different cases are analysed in Fig. 25, corresponding to three input signals from M-VCSEL (reproducing the signals from photoreceptors in the eye) with stimulus duration of $3.6 \mathrm{~ns}, 5.18 \mathrm{~ns}$ and $6.22 \mathrm{~ns}$ respectively. Fig. 25(a) (plot (i), in black) shows results when 3.6ns-long stimuli from M-VCSEL arrive into BC-VCSEL. Fig. 25(a) (plot (ii), in red) shows the times series from BC-VCSEL, which yields initially a lower constant output power level until 
it produces a positive graded potential in response to an arriving stimulus (marked by a solid black line). Plot (iii) (in blue) in Fig. 25(a) shows the time series measured at RGCVCSEL's output. This output is characterised by a continuous (tonic) spike firing response, which is fully inhibited upon the arrival of the positive graded potential from BC-VCSEL (marked by a solid black line). Figs. 25(b) and 25(c) plot results for the two other cases of stimulus duration studied, namely $5.18 \mathrm{~ns}$ (Fig. 25(b)) and $6.22 \mathrm{~ns}$ (Fig. 25(c)). In both cases, BC-VCSEL produces a positive graded potential in response to the incoming perturbation (solid back lines in the plots) which is translated into a spiking inhibition window at RGC-VCSEL's output for the duration of the graded potentials (5.18 and $6.22 \mathrm{~ns}$ respectively). Hence, as Figs. 25(a-c) show, simply by controlling the stimulus' length the window of spike inhibition achieved at the output of the OFFtype $\mathrm{BC}+\mathrm{RGC}$ circuit can be tuned at will. This is further illustrated in the temporal maps included in Fig. 26 that plot the results obtained at the output of the OFF-type BC+RGC system (RGC-VCSEL's output) for the three cases investigated in Fig. 25 when 60 consecutive stimuli from MVCSEL are launched into BC-VCSEL. For the 3 cases, every single one of the 60 stimuli trigger analogous spike inhibition windows (of different temporal length depending on the case of study) at the circuit's output.

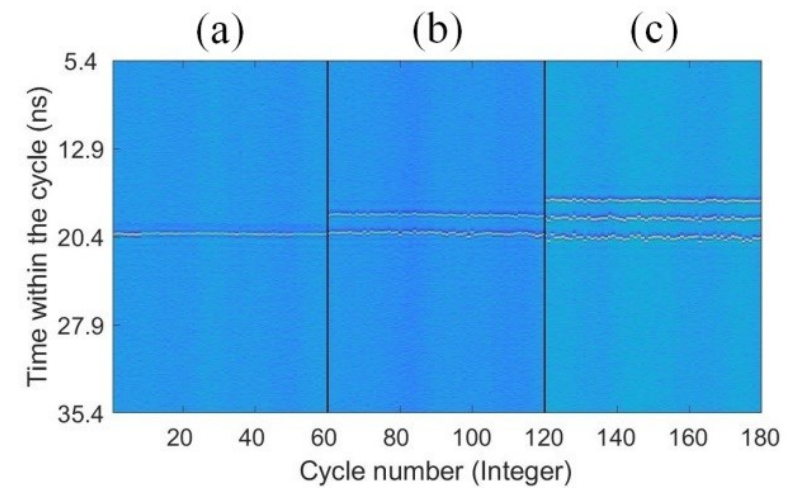

Fig. 24. Temporal maps measured at the RGC-VCSEL output in the ON-type $\mathrm{BC}+\mathrm{RGC}$ circuit. Each map plots the system's response to the arrival of 60 consequetive input stimuli from M-VCSEL for the three cases of stimulus duration analysed in Fig. 23, namely (a) 0.85 ns, (b) $2.79 \mathrm{~ns}$ and (c) $4.90 \mathrm{~ns}$.

Figs. 23-26 demonstrate the controllability and reproducibility of the obtained responses from the ON- and OFF-type retinal neuronal circuits emulated with VCSEL-Neurons. Moreover, it should be stated that the results in Figs. 23-26, also reveal the ultrafast photonic emulation of the selected retinal neuronal circuits depicted graphically in Fig. 20. In all cases, sub-ns spiking signals and sub-ns/ns graded potential signals are used for operation in the photonic emulation systems. These are $>7$ orders of magnitude faster than the millisecond timescales in the emulated biological neuronal retinal circuits. Further, devices operating at telecom wavelengths $(1550 \mathrm{~nm})$ are used in this new research direction. These results therefore open exciting prospects for ultrafast and practical emulation of complex interconnected neuronal circuits/networks for alternative simulation platforms to help deepen our understanding of brain circuit activitiy as well as for future new paradigms in neuro-inspired computing, communication, data processing and Artificial Intelligence.
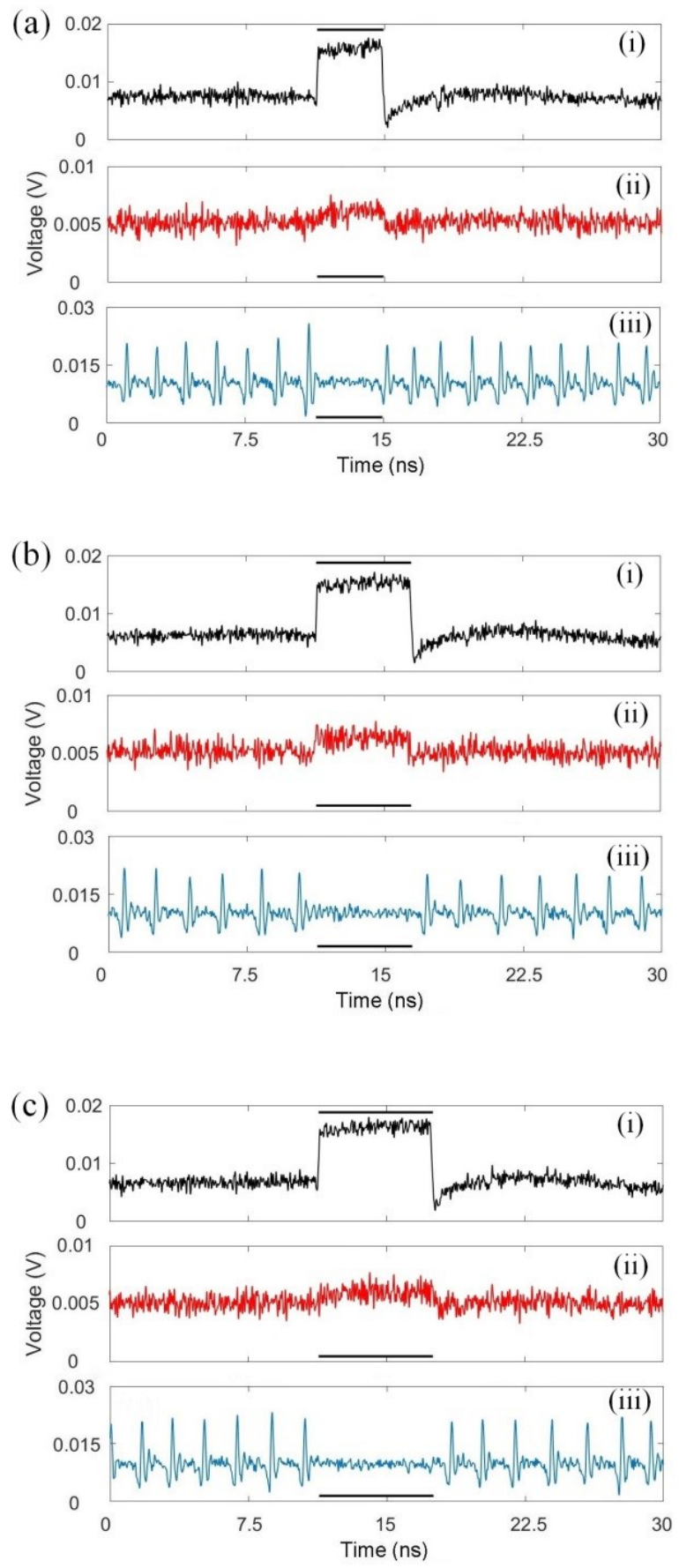

Fig. 25. Photonic emulation of an OFF-type BC+RGC circuit with VCSELNeurons. Three cases are investigated showing results for M-VCSEL ((i), in black) input signals with different stimulus lengths of (a) $3.60 \mathrm{~ns}$, (b) $5.18 \mathrm{~ns}$ and (c) $6.22 \mathrm{~ns}$. Plots (ii) (in red) depict the measured time series from BCVCSEL. This reacts by producing graded potentials in response to the input stimuli. Plots (ii) (in blue) show the measured time series from RGC-VCSEL. This yields a tonic spiking output, until a graded potentials arrives from BCVCSEL inhibiting the spiking response from RGC-VCSEL. VCSELs operating at $1550 \mathrm{~nm}$ were used in this work. The M-, BC- and RGC-VCSELs were driven with bias currents of $4.61 \mathrm{~mA}$ (at $298 \mathrm{~K}$ ), $4.3 \mathrm{~mA}$ (at $290 \mathrm{~K}$ ) and $3.72 \mathrm{~mA}$ (at $300 \mathrm{~K}$ ) respectively to tune their emission wavelengths. The injected signals from M-VCSEL into BC-VCSEL were set with a constant power level of $57.5 \mu \mathrm{W}$ (with added $78.6 \mu \mathrm{W}$ power raises) and an initial frequency detuning of $\Delta \mathrm{f}_{\mathrm{BC}-\mathrm{M}}=-4.81 \mathrm{GHz}$. The injection from BC-VCSEL into RGC-VCSEL was set with a constant power level of $8.8 \mu \mathrm{W}$ and a frequency detuning of $\Delta \mathrm{f}_{\mathrm{RGC}-\mathrm{BC}}=-4.81 \mathrm{GHz}$. 
(a)

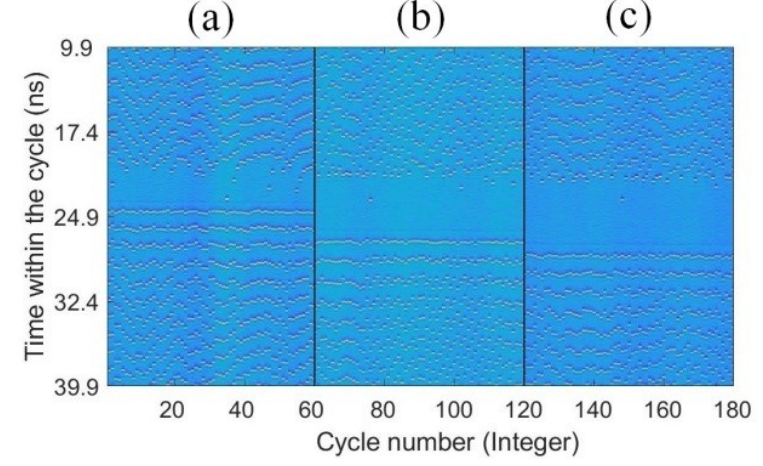

Fig. 26. Temporal maps measured at the RGC-VCSEL output in the OFF-type $\mathrm{BC}+\mathrm{RGC}$ circuit. Each map plots the system's response to the arrival of 60 consequetive input stimuli from M-VCSEL for the three cases of stimulus' duration analysed in Fig. 23, namely (a) $3.60 \mathrm{~ns}$, (b) $5.18 \mathrm{~ns}$ and (c) $6.22 \mathrm{~ns}$.

\section{CONCLUSION}

In summary, in this work we report on ultrafast artificial optical neurons based upon VCSELs towards future laser neuron network implementations for use in the emerging field of neuromorphic photonics. We introduce our recent as well as our currently ongoing work demonstrating experimentally that controllable spiking responses, analogous to those obtained in biological neurons, including spiking excitation and inhibition can be obtained at sub-ns speed rates with VCSEL-Neurons. These sub-ns spiking regimes are achieved in response to external perturbations (stimuli) which can be encoded either in the optical or electronic inputs of the VCSEL-Neurons. Moreover, the spiking dynamical regimes are achieved at speed rates $>7$ orders of magnitude faster than the millisecond timescales of biological neurons. Our results also show that VCSEL-Neurons can exhibit multiple neuronal responses in addition to emulating excitatory and inhibitory neurons, including among others, phasic and tonic spiking, thresholding and stimulus information encoding in the spike latency or spike firing rate. We also report new experimental results demonstrating the practical development of ultrafast spiking processing systems based upon VCSEL-Neurons. These include spiking memory modules, using either single or mutually-coupled VCSEL-Neurons, and which are able to store complex patterns of (sub-ns) spikes within in-built delayed feedback (or mutual-coupling) loops. Additionally, we experimentally investigate the potentials of VCSELNeurons for the photonic emulation of neuronal circuits in the retina at ultrafast speeds. Specifically, we demonstrate the photonic emulation of ON-/OFF-type Bipolar Cell and Retinal Ganglion Cell circuits in the retina performing pre-processing of incoming visual information from photoreceptors in the eye, before transmitting (via the optic nerve) converted spiking signals to the visual cortex in the brain for further processing. Finally, all the results presented in this work are obtained with commercially available inexpensive VCSELs operating at the telecom wavelengths of $1310 \mathrm{~nm}$ and 1550 $\mathrm{nm}$; hence fully compatible with current optical telecommunication network and data center technologies. This opens exciting prospects for new functional ultrafast neuromorphic photonic systems based on VCSEL-Neurons and laser-neuron networks for novel paradigms in braininspired information processing, computing and Artificial Intelligence.

\section{ACKNOWLEDGMENT}

The authors wish to thank Dr Julian Javaloyes from Universitat Illes Balears (Spain) and Prof. Tao Deng from Southwest University Chongqing (China).

\section{REFERENCES}

[1] C. Mead, "Analogue VLSI and Neural Systems," Addison-Wesley (1989).

[2] B. Benjamin, P. Gao, E. McQuinn, et al., "Neurogrid: A mixed-analogdigital multichip system for large-scale neural simulations," Proc. IEEE, vol. 102, pp. 699-716, (2014).

[3] J. Schemmel, D. Brüderle, A. Grübl, et al., "A wafer-scale neuromorphic hardware system for large-scale neural modeling," in Proc. IEEE International Symposium on Circuits and Systems, pp. 1947-1950, (2010).

[4] P. A. Merolla, J. V. Arthur, R. Alvarez-Icaza, et al., "A million spikingneuron integrated circuit with a scalable communication network and interface," Science, vol. 345, pp. 668-672, (2014).

[5] S. Furber, F. Galluppi, S. Temple, and L. Plana, "The SPiNNaker project," Proc. IEEE, vol. 102, pp. 652-665, (2014).

[6] E. C. Mos, J. L. Hoppenbrouwers, M. T. Hill, et al., "Optical neuron by use of a laser diode with injection seeding and external optical feedback," IEEE Trans. Neural Netw., vol. 11, pp. 988-996, (2000).

[7] P. R. Prucnal and B. J. Shastri, Neuromorphic Photonics, 1 ed., CRC Press, (2017).

[8] A. M. Yacomotti, P. Monnier, F. Raineri, et al., "Fast thermo-optical excitability in a two-dimensional photonic crystal," Phys. Rev. Lett., vol. 97, 143904, (2006).

[9] M. Brunstein, A. M. Yacomotti, I. Sagnes, et al., "Excitability and selfpulsating in a photonic crystal nanocavity," Phys. Rev. A, vol. 85, 031803, (2012).

[10] B. Romeira, J. Javaloyes, C. N. Ironside, et al., "Excitability and optical pulse generation in semiconductor lasers driven by resonant tunneling diode photodetectors," Opt. Exp., vol. 21, pp. 20931-20940, (2013).

[11] B. Romeira, J. M. L. Figueiredo and J. Javaloyes, "Delay dynamics of neuromorphic optoelectronic nanoscale resonators: perspectives and applications," Chaos, vol. 27, 114323, (2017).

[12] B. J. Shastri, M. A. Nahmias, A. N. Tait, et al., "SIMPEL: circuit model for photonic spike processing laser neurons," Opt. Exp., vol. 23, pp. 8029-8044, (2015).

[13] B. J. Shastri, M. A. Nahmias, A. N. Tait, et al., "Spike processing with a graphene excitable laser," Sci. Rep., vol. 6, 19126, (2016).

[14] K. Kravtsov, M. P. Fok, D. Rosenbluth, and P. R. Prucnal, "Ultrafast alloptical implementation of a leaky integrate-and-fire neuron," Opt. Exp., vol. 19, pp. 2133-2147, (2011).

[15] R. Toole, A. N. Tait, T. Ferreira de Lima, et al., "Photonic implementation of spike-timing-dependant plasticity and learning algorithms of biological neural systems," J. Lightwave Technol., vol. 34, pp. 470-476, (2016).

[16] A. Tait, T. Ferreira de Lima, E. Zhou, et al., "Neuromorphic photonic networks using silicon photonics weight banks," Sci. Rep., vol. 7, 7430, (2017).

[17] M. P. Fok, Y. Tian, D. Rosenbluth, and P. R. Prucnal, "Asynchronous spiking photonic neuron for lightwave neuromorphic signal processing," Opt. Lett., vol. 37, pp. 3309-3311, (2012).

[18] K. Alexander, T. Van Vaerenbergh, M. Fiers, et al., "Excitability in optically injected microdisk lasers with phase controlled excitatory and inhibitory response," Opt. Exp., vol. 21, pp. 26182-26191, (2013).

[19] S. Barbay, R. Kuszelewicz, and A. M. Yacomotti, "Excitability in a semiconductor laser with saturable absorber," Opt. Lett., vol. 36, 44764478, (2011).

[20] F. Selmi, R. Braive, G. Beaudoin, et al., "Relative Refractory Period in an Excitable Semiconductor Laser," Phys. Rev. Lett., vol. 112, 183902, (2014).

[21] F. Selmi, R. Braive, G. Beaudoin, et al., "Spike latency and response properties of an excitable micropillar laser," Phys. Rev. E, vol. 94, 042219, (2016). 
[22] W. Coomans, L. Gelens, S. Beri, et al., "Solitary and coupled semiconductor ring lasers as optical spiking neurons," Phys. Rev. E, vol. 84, 036209, (2011).

[23] L. Gelens, L. Mashal, S. Beri, et al., "Excitability in semiconductor microring lasers: Experimental and theoretical pulse characterization," Phys. Rev. A, vol. 82, 063841, (2010).

[24] C. Mesaritakis, A. Kapsalis, A. Bogris and D. Syvridis, "Artificial neuron based on integrated semiconductor quantum dot mode-locked lasers," Sci. Rep., vol. 6, 39317, (2016).

[25] B. Kelleher, C. Bonatto, G. Huyet, and S. P. Hegarty, "Excitability in optically injected semiconductor lasers: Contrasting quantum-well- and quantum-dot-based device," Phys. Rev. E, vol. 83, 026207, (2011).

[26] B. Kelleher, C. Bonatto, P. Skoda, et al., "Excitation regeneration in delay-coupled oscillators," Phys. Rev. E, vol. 81, 036204, (2010).

[27] M. A. Nahmias, B. J. Shastri, A. N. Tait and P. R. Prucnal, "A leaky integrate-and-fire laser neuron for ultrafast cognitive computing," IEEE J. Sel. Top. Quantum Electron., vol. 19, 1800212, (2013).

[28] A. Hurtado, K. Schires, I. D. Henning and M. J. Adams, "Investigation of vertical cavity surface emitting laser dynamics for neuromorphic photonic systems," Appl. Phys. Lett., vol. 100, 103703, (2012).

[29] M. Turconi, B. Garbin, M. Feyereisen, et al., "Control of excitable pulses in an injection-locked semiconductor laser," Phys. Rev. E, vol. 88, 022923, (2013).

[30] B. Garbin, J. Javaloyes, G. Tissoni and S. Barland, "Topological solitons as addressable bits in a driven laser," Nat. Commun., vol. 6, 5915, (2015).

[31] A. Dolcemascolo, B. Garbin, B. Peyce, R. Veltz and S. Barland, "Resonator neuron and triggering multipulse excitability in laser with injected signal," Phys. Rev. E, vol. 98, 062211, (2018).

[32] S. Xiang, A. Wen and W. Pan, "Emulation of spiking response and spiking frequency property in VCSEL-based photonic neuron," IEEE Photon. J., vol. 8, 1504109, (2016).

[33] P. R. Prucnal, B. J. Shastri and T. Ferreira de Lima, "Recent progress in semiconductor excitable lasers for photonic spike processing," Adv. Opt. Photon., vol. 8, pp. 228-299, (2016).

[34] B. J. Shastri, A. N. Tait, T. Ferreira de Lima, et al., Principles of neuromorphic photonics. Encyclopedia of complexity and system science, Berlin, Heidelberg, Springer, (2018).

[35] A. Hurtado, I. D. Henning and M. J. Adams, "Optical neuron using polarization switching in a $1550 \mathrm{~nm}$-VCSEL," Opt. Exp., vol. 18, pp. 25170-25176, (2010).

[36] P. Pérez, A. Valle, L. Pesquera and A. Quirce, "All-optical inverter based on polarization switching in VCSELs subject to single and dual optical injection," IEEE J. Sel. Top. Quantum Electron., vol. 19, 1700408, (2012).

[37] A. Hurtado and J. Javaloyes, "Controllable spiking patterns in longwavelength vertical cavity surface emitting lasers for neuromorphic photonics systems," Appl. Phys. Lett., vol. 107, 241103, (2015).

[38] J. Robertson, E.Wade and A. Hurtado, "Electrically controlled neuronlike spiking regimes in vertical-cavity surface-emitting lasers at ultrafast rates", IEEE J. Sel. Top. Quantum Electron., vol. 25, 5100307, (2019).

[39] J. Robertson, T. Deng, J. Javaloyes and A. Hurtado, "Controlled inhibition of spiking dynamics in VCSELs for neuromorphic photonics: theory and experiments," Opt. Lett., vol. 42, 1560-1563, (2017).

[40] T. Deng, J. Robertson and A. Hurtado, "Controlled propagation of spiking dynamics in vertical-cavity surface-emitting lasers: towards neuromorphic photonic networks," J. Sel. Top. Quantum Electron., vol. 23, 1800408, (2017).

[41] T. Deng, J. Robertson, Z. M. Wu, et al., "Stable propagation of inhibited spiking dynamics in vertical-avity surface-emitting lasers for neuromorphic photonics networks," IEEE Access, vol. 6, pp. 6795167958, (2018).

[42] A. Dolcemascolo, A. Miazek, F. Marino, R. Veltz, S. Barland, "Meanfield dynamics of a fully connected network of spiking neuromorphic semiconductor lasers," European Semiconductor Laser Workshop 2018, pp. 9-10, Sep (2018)

[43] S. Xiang, Y. Zhang, X. Guo, et al., "Photonics generation of neuron-like dynamics using VCSELs subject to double polarized optical injection," J. Lightwave Technol., vol. 36, pp. 4227-4234, (2018).

[44] Y. Zhang, S. Xiang, X. Guo, et al., "Polarization-resolved and polarization-multiplexed spike encoding properties in photonic neuron based on VCSEL-SA," Sci. Rep., vol. 8, 16095, (2018).

[45] S. Xiang, H. Zhang, X. Guo, et al., "Cascadable neuron-like spiking dynamics in coupled VCSELs subject to orthogonally polarized optical pulse injection,” J. Sel. Top. Quantum Electron., vol. 23, 1700207, (2017).

[46] S. Xiang, J. Gong, Y. Zhang, X. Guo, Y. Han, A. Wen, Y, Hao, "Numerical implementation of wavelength-dependent photonic spike timing dependent plasticity based on VCSOA,", IEEE J. Quantum Electron., vol. 54 , 8100107 (2018).

[47] Y. Zhang, S. Xiang, J. Gong, et al., "Spike encoding and storage properties in mutually coupled vertical-cavity surface-emitting lasers subject to optical pulse injection," Appl. Opt., vol. 57, pp. 1731-1737, (2018).

[48] Y. Zhang, S. Xiang, X. Guo, A. Wen and Y. Hao, "All-optical inhibitory dynamics in photonic neuron based on polarization mode competition in a VCSEL with an embedded saturable absorber," Opt. Lett., vol. 44, pp. 1548-1551, (2019).

[49] S. Xiang, Y. Zhang, J. Gong, X. Guo, L. Lin and Y. Hao, "STDP-based unsupervised spike pattern learning in a photonic spiking neural network with VCSELs and VCSOAs," IEEE J. Sel. Top. Quantum Electron., vol. 25, 1700109, (2019).

[50] K. Schires, A. Hurtado, I. D. Henning and M. J. Adams, "Rare disruptive events in polarization-resolved dynamics of optically injected 1550 nm VCSELs," Electron. Lett., vol. 48, pp. 872-874, (2012).

[51] R. Al-Seyab, K. Schires, N.A. Khan, A. Hurtado, I.D. Henning and M.J. Adams, "Dynamics of polarized optical injection in 1550-nm VCSELs: theory and experiments," IEEE J. Sel. Top. Quantum Electron., vol. 17, 1242-1249 (2011).

[52] K. Al-Naimee, F. Marino, M. Ciszak, et al., "Excitability of periodic and chaotic lasers with optoelectronic feedback," Eur. Phys. J. D, vol. 58, pp. 187-189, (2010).

[53] H. J. Wunsche, O. Brox, M. Radziunas, and F. Henneberger, "Excitability of a semiconductor laser by a two-mode homoclinic bifurcation," Phys. Rev. Lett., vol. 88, 023901, (2002).

[54] J. P. Toomey, C. Nichkawade, D. M. Kane, et al., "Stability of the nonlinear dynamics of an optically injected VCSEL," Opt. Exp., vol. 20, pp. 10256-10270, (2012).

[55] A. Hurtado, A. Quirce, A. Valle, et al., "Nonlinear dynamics induced by parallel and orthogonal optical injection in $1550 \mathrm{~nm}$ vertical-cavity surface-emitting lasers (VCSELs)," Opt. Exp., vol. 18, pp. 9423-9428, (2010).

[56] M. J. Adams, A. Hurtado, D. Labukhin and I. D. Henning, "Non-linear semiconductor lasers and amplifiers for all-optical information processing," Chaos, vol. 20, 037102, (2010).

[57] F. Koyama, "Recent advances of VCSEL photonics," J. Lightwave Technol., vol. 24, pp. 4502-4513, (2006).

[58] E. M. Izhikevich, "Which model to use for cortical spiking neurons," IEEE Trans. Neural Netw. 15, 1063 (2004).

[59] B. Kelleher, C. Bonatto, P. Skoda, S. P. Hegarty and G. Huyet, "Excitation regeneration in delay-coupled oscillators," Phys. Rev. E, vol. 81, 036204, (2010).

[60] C.A. Mead and M.A. Mahovald, "A silicon model of early visual processing," Neural Networks, vol. 1, 91-97 (1988).

[61] C.A. Mead, "Neuromorphic electronic systems," Proc. IEEE, vol. 78, pp. 1629-1636 (1990).

[62] M.A. Mahowald, C.A. Mead, "The silicon retina," Sci. Am., vol. 264, 76-82 (1991).

[63] C. Posch, T. Serrano-Gotarredona, B. Linares-Barranco, T. Delbruck, "Retinomorphic event-based vision sensors: bioinspired cameras with spiking output," Proc. IEEE, vol. 102, 1470-1484 (2014).

[64] P. Lichtsteiner, T. Delbruck, "A $64 \times 64$ AER logarithmic temporal derivative silicon retina", Res. Microelectron. Electron., vol. 2, 202-205 (2005).

[65] T. Serrano-Gotarredona, B. Linares-Barranco, "A $128 \times 128 \quad 1.5 \%$ contrast sensitivity $0.9 \%$ FPN $3 \mu$ s latency $4 \mathrm{~mW}$ asynchronous framefree dynamic vision sensor using transimpedance preamplifiers", IEEE J. Solid-State Circ., vol. 48, 827-838 (2013).

[66] T. Euler, S. Hverkamp, T. Schubert and T. Baden, "Retinal bipolar cells: elementary building blocks of vision," Nat. Rev. Neuroscience, vol. 15, 507-519 (2014).

[67] R.H. Masland, "The neuronal organization of the retina", Neuron, vol. 76, 266-280 (2012).

[68] E.R. Kandel, J.H. Schwartz, T.M. Jessell, "Principles of Neural Science,"5th ed. McGraw-Hill, New York, (2012). 
Joshua Robertson was born in Glasgow, U.K. in 1994. He received his MPhys degree in Physics with specialization in Photonics from the University of Strathclyde, Glasgow in 2017. He is currently working towards his Ph.D. degree at the Institute of Photonics, University of Strathclyde, Glasgow, focusing on neuromorphic photonic systems with lasers.

Ewan Wade was born in Glasgow, U.K. in 1996. He is currently working towards his MPhys degree in Physics with specialization in Photonics from the University of Strathclyde, Glasgow.

Yasmin Kopp was born in Hamburg, Germany in 1995. She is currently working towards her degree in Physics from Westfälische-Wilhelms-Universität Münster, Münster and the University of Strathclyde, Glasgow.

Julian Bueno was born in Palma de Mallorca, Spain in 1988. He is currently a Research Associate at the Institute of Photonics, University of Strathclyde, Glasgow, working in the 'BRAIN LASER' project funded by ONRG to develop novel neuromorphic photonic systems and networks with laser-based optical neurons.

Antonio Hurtado received the Ph.D. degree from the Universidad Politécnica de Madrid (UPM), Madrid, Spain in December 2006. He has more than 10 years' international research experience in photonics in the UK (Universities of Essex and Strathclyde), USA (University of New Mexico), and Spain (UPM). He received two Marie Curie Fellowships by the European Commission: Projects ISLAS (2009-2011) \& NINFA (2011-2014). In 2014 he was awarded Chancellor's Fellowship by the University of Strathclyde following which he was appointed as a Lecturer (Assist. Prof.) in the Strathclyde's Institute of Photonics (Physics Dept.). He was promoted to Senior Lecturer (Assoc. Prof.) in 2018. His current research interests are but not limited to neuromorphic photonics, laser nonlinear dynamics, nanolaser systems and hybrid nanofabrication. 